\title{
Neuroleptics as therapeutic compounds stabilizing neuromuscular transmission in amyotrophic lateral sclerosis
}

\author{
Shunmoogum A. Patten, ${ }^{1,2,3}$ Dina Aggad, ${ }^{1,2,4}$ Jose Martinez, ${ }^{5}$ Elsa Tremblay, ${ }^{1,6}$ Janet Petrillo, ${ }^{5}$ \\ Gary A.B. Armstrong, ${ }^{1,2,7}$ Alexandre La Fontaine, ${ }^{1,6}$ Claudia Maios, ${ }^{1,2}$ Meijiang Liao, ${ }^{1,2}$ Sorana Ciura, ${ }^{8}$ \\ Xiao-Yan Wen, ${ }^{9}$ Victor Rafuse, ${ }^{10}$ Justin Ichida, ${ }^{11}$ Lorne Zinman, ${ }^{12}$ Jean-Pierre Julien, ${ }^{13}$ Edor Kabashi, ${ }^{8}$ \\ Richard Robitaille, ${ }^{1,6}$ Lawrence Korngut, ${ }^{5}$ J. Alexander Parker, ${ }^{1,2}$ and Pierre Drapeau, ${ }^{1,2,6}$ \\ 'Department of Neuroscience, Université de Montréal, Montréal, Canada. ${ }^{2}$ Centre de recherche du centre hospitalier de \\ I'Université de Montréal (CRCHUM), Montréal, Canada. ${ }^{3}$ INRS Institut Armand-Frappier, Laval, Canada. ${ }^{4}$ Institut des \\ Biomolécules Max Mousseron IBMM, UMR 5247, CNRS-Université Montpellier-ENSCM, Montpellier, France. ${ }^{5}$ Department \\ of Clinical Neurosciences, Hotchkiss Brain Institute, University of Calgary, Alberta, Canada. ${ }^{6} \mathrm{FRQS}$ Groupe de recherche \\ sur le système nerveux central, Montreal, Canada. 'Department of Neurology and Neurosurgery, McGill University and \\ Montreal Neurological Institute, Montreal, Canada. ${ }^{8}$ Sorbonne Université, Université Pierre et Marie Curie (UPMC), \\ Université de Paris 06, Institut du Cerveau et de la Moelle Épinière (ICM), Paris, France. ${ }^{9}$ Zebrafish Centre for Advanced \\ Drug Discovery \& Keenan Research Centre for Biomedical Science, Li Ka Sheng Knowledge Institute, St. Michael's Hospital \\ and Department of Medicine \& Physiology, University of Toronto, Toronto, Ontario, Canada. ${ }^{10}$ Department of Medical \\ Neuroscience, Dalhousie University, Halifax, Nova Scotia, Canada. "Department of Stem Cell Biology and Regenerative \\ Medicine, Keck School of Medicine and Eli and Edythe Broad Center for Regenerative Medicine and Stem Cell Research, \\ University of Southern California, Los Angeles, California, USA. ${ }^{2}$ Department of Neurology, University of Toronto, \\ Sunnybrook Health Sciences Centre,Toronto, Ontario, Canada. ${ }^{13}$ Centre de recherche CERVO, Chemin de la Canardière, \\ Université Laval, Québec City, Canada.
}

Amyotrophic lateral sclerosis (ALS) is a rapidly progressing, fatal disorder with no effective treatment. We used simple genetic models of ALS to screen phenotypically for potential therapeutic compounds. We screened libraries of compounds in C. elegans, validated hits in zebrafish, and tested the most potent molecule in mice and in a small clinical trial. We identified a class of neuroleptics that restored motility in C. elegans and in zebrafish, and the most potent was pimozide, which blocked T-type $\mathrm{Ca}^{2+}$ channels in these simple models and stabilized neuromuscular transmission in zebrafish and enhanced it in mice. Finally, a short randomized controlled trial of sporadic ALS subjects demonstrated stabilization of motility and evidence of target engagement at the neuromuscular junction. Simple genetic models are, thus, useful in identifying promising compounds for the treatment of ALS, such as neuroleptics, which may stabilize neuromuscular transmission and prolong survival in this disease.

Authorship note: SAP and DA are co-first authors. LK, JAP, and PD are co-senior authors.

Conflict of interest: The authors have declared that no conflict of interest exists.

Submitted: August 28, 2017 Accepted: October 17, 2017 Published: November 16, 2017

Reference information: JCI Insight. 2017;2(22):e97152. https://doi.org/10.1172/jici. insight.97152.

\section{Introduction}

Amyotrophic lateral sclerosis (ALS) is a debilitating and fatal neurodegenerative disorder affecting motor function, with mean survival of 3 years from disease onset. Considerable progress has been made in unraveling the genetic etiology of ALS. This led to the development of animal models that permitted significant pathobiological insights and led to tremendous efforts that have so far unfortunately failed to find a treatment or cure for ALS (1). The treatments to slow the progression of ALS are riluzole (2) and more recently edaravone (3), but they are only modestly effective. Therefore, there is an urgent need to develop and assess new therapeutics for ALS, which remains one of the greatest challenges in neurology. Here, we consider neuromuscular junction (NMJ) transmission as a potential therapeutic target and biomarker.

Clinical and electrophysiological correlates of muscle fatigability suggesting an element of NMJ dysfunction have been described in patients with ALS over the past 50 years (4). Prior reports of decrement on repetitive nerve stimulation (RNS) corrected by neostigmine in poliomyelitis supported the hypothesis 
of NMJ dysfunction accompanying cell death and axonal degeneration of the lower motor neurons (5). Decremental responses of the trapezius were found in $29 \%$ of 129 patients with ALS that were significantly improved by postexercise facilitation and edrophonium in combination but not alone (6). Recent work has demonstrated more prevalent decremental responses in the muscles of the thenar eminence (thumb abductor pollicis brevis [APB]) compared with the hypothenar eminence (abductor digit minimi $[\mathrm{ADM}]$ ) correlating with the classic dissociation of involvement of the lateral more than medial hand in ALS (7).

The locus of the clinical deficit at the NMJ has been gleaned from electrophysiological studies. Decremental responses in ALS have different characteristics from classic presynaptic (Lambert-Eaton Myasthenic Syndrome) and postsynaptic (myasthenia gravis) disorders of the NMJ (6). The authors observed that initial compound motor action potentials (CMAP) were of low amplitude but did not demonstrate early postexercise facilitation and concluded that low baseline CMAP amplitudes with decrement might suggest a presynaptic transmission deficit (8). Reinnervation of motor endplates with reduced acetylcholine (ACh) stores could cause decremental responses, and in vitro microelectrode studies of the anconeus muscle in ALS subjects demonstrated reduced presynaptic ACh quantal stores, possibly explained by the diminished size of nerve terminals (8).

NMJ dysfunction has also been found in animal models of ALS, such that it is now widely recognized that extensive nerve sprouting and synaptic remodeling occur at the NMJ long before presentation of symptoms (9). In mutant SOD1-expressing mice (10), an early retraction or "die-back" of presynaptic motor endings was observed long before the death of motoneurons (11), and this has also been observed in TARDBP mice (12) and in tissue from patients with ALS (13). Expression of either mutant human TARDBP (14) or FUS mRNA in zebrafish (15) resulted in impaired transmission reduced frequency of miniature endplate currents (mEPCs) and reduced quantal transmission at the NMJ. Expression in muscle of a variety of trophic factors in SOD1 mice has been tested for their ability to stabilize the NMJ (16), but these treatments have shown only limited success in mice and humans $(17,18)$. This is possibly because they have addressed NMJ die-forward as a consequence of motoneuron degeneration rather than die-back as a consequence of earlier motoneuron deficits (19). Increased NMJ denervation (possibly due to die-back) has also been reported in rat and mouse models of TDP-43 mutations $(12,20)$. Treatments directed toward motoneuron function, thus, remain an interesting and largely unexplored approach to prevent or delay disease onset and progression. Therefore, screening for small molecules that can restore or ameliorate motility and NMJ transmission could identify novel ALS therapeutics.

Simple animal models of ALS are powerful tools for therapeutic discovery of compounds that suppress disease phenotype, regardless of the specific molecular targets (21). We previously developed C. elegans (22) and zebrafish (23) genetic models of ALS that are amenable to testing compounds that can preserve motility and reduce neurodegeneration as an independent way of discovering potential therapeutic agents $(24,25)$. The goal of the current study was to use our drug-screening platform to screen large numbers of compounds in order to identify small molecules with acute effects that could be immediately translated into the clinic using short-term improvement of motor function as a read-out. We screened 3,850 clinically approved compounds and identified a class of neuroleptics that stabilized motility in $C$. elegans, zebrafish, and mice expressing human genes with mutations in ALS, and we tested our lead compound pimozide in a small phase II randomized placebo-controlled randomized clinical trial (RCT) in subjects with ALS. Our findings indicate that pimozide, and potentially other neuroleptics and agents acting at the NMJ, may prove promising as an ALS therapeutic to stabilize motor activity and prolong survival.

\section{Results}

\section{Chemical genetic screen in C. elegans and zebrafish genetic models of ALS}

We performed a high-throughput screen of 3,850 small molecules in the (partially overlapping) Biomol Natural Products, MicroSource Discovery Spectrum, Prestwick Commercial Products, and Sigma LOPAC sets in $C$. elegans transgenics that express mutant TDP-43 (TARDBPA315T, referred to herein as mTDP-43). These worms exhibit paralysis phenotypes in a matter of hours when grown in liquid culture (22), and we identified 24 compounds capable of rescuing paralysis when tested for 6 hours at a concentration of $20 \mu \mathrm{M}$ : 11 were excluded as being irrelevant (insecticides, antibacterial drugs, and antitumor drugs), whereas the other 13 compounds were considered as positive hits and, interestingly, were all neuroleptics (Figure 1): mianserin, amoxapine, nicergoline, kawain, pimethixene, methiotepin, octoclopethin, flupentixol, chlorprothixene clozapine, pizotifen, cyproheptadine, and pimozide. 


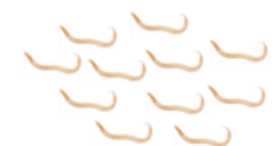

mTDP-43 C. elegans

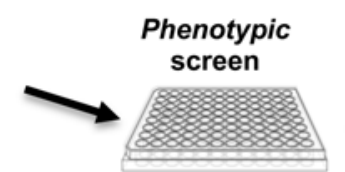

Chemical libraries
\begin{tabular}{|l|}
\hline Microsource Discovery \\
Sigma Lopac \\
Prestwick \\
Biomol \\
\hline
\end{tabular}

\section{0 compounds}

Clozapine
Mianserine
Amoxapine
Cyproheptadine
Nicergoline
Kawain
Pimethixene
Pimozide
Pizotifen
Methiothepin
(士)-Octoclothepin
Flupentixol
Chlorprothixene

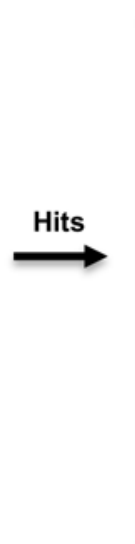

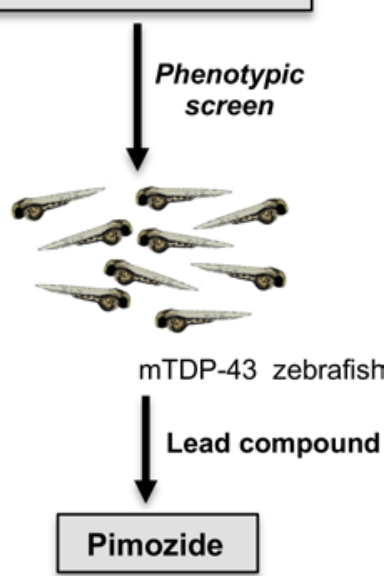

Figure 1. Chemical genetic screen identified 13 compounds as neuroprotective in models of ALS. Chemical libraries (3,850 compounds) were first screened in a C. elegans TDP-43 model, and positive hits were tested on zebrafish models of ALS. Individual or small numbers of animals were transferred into multiwell dishes in which different test compounds were present and incubated for 6 hours (C. elegans) or overnight (zebrafish). We identified 13 compounds that improved motor behavior (motility) phenotypes in mutant TDP-43 (mTDP43) C. elegans. These positive hits were then tested on MTDP-43 zebrafish at various concentrations (1-20 $\mu \mathrm{M})$. We identified 10 active compounds (highlighted in bold) in our zebrafish model, and the most potent compound was found to be pimozide, which was active at $1 \mu \mathrm{M}$.

Positive hits were then retested in worms at concentrations ranging from $2-40 \mu \mathrm{M}$ and validated in our zebrafish vertebrate model. Zebrafish expressing the analogous mutant TDP-43 (TARDBPG348C, referred to herein as mTDP-43) have impaired swimming as assessed by their limited ability to produce a touchevoked escape response (23). We confirmed 10 active compounds (Figure 1, shown in bold) in zebrafish upon overnight treatment and identified pimozide as the most potent lead compound (Figure 1).

The neuropletic pimozide as lead neuroprotective compound

As motoneuron death occurs at the terminal stage of ALS, we examined the effects of our lead compound pimozide on motoneuron degeneration in our models. Progressive paralysis phenotypes in transgenic $C$. elegans expressing mTDP-43 in motor neurons were accompanied by neurodegeneration that was age-dependent and occurred at a higher rate at day 9 when grown on plates. We then retested pimozide in worms on plates over this period at a range of concentrations $(2 \mu \mathrm{M}, 20 \mu \mathrm{M}$, and $40 \mu \mathrm{M})$ and assessed their motor phenotypes, including motoneuron degeneration. We found that pimozide significantly prevented the paralysis phenotype in the treated mTDP-43 worms (Figure $2 \mathrm{~A}, P<0.05$ ), and the neurodegenerative phenotypes - marked as breaks along neuronal processes (22) - were also significantly reduced by treatment with pimozide (Figure $2 \mathrm{~B}, P<0.05$ ).

To confirm the neuroprotective properties of pimozide during acute treatment, we tested it overnight at a range of concentrations $(0.5 \mu \mathrm{M}, 1 \mu \mathrm{M}, 2 \mu \mathrm{M}, 5 \mu \mathrm{M}$, and $10 \mu \mathrm{M})$ in our zebrafish model of mTDP43 toxicity, which shows motor behavioral and axonal phenotypes (23). Zebrafish expressing mTDP-43 showed a greatly reduced touch-evoked escape response compared with wtTDP-43 fish (Figure 2C). Interestingly, mTDP-43 fish treated with $1 \mu \mathrm{M}$ pimozide, the lowest effective dose observed, showed an improved swimming response, as assessed by the distance swam, swim duration, and maximum swim velocity (Figure 2, D-F, $P<0.05$ ). We then sought to examine the motor axonal phenotypes following treatment with pimozide. Immunohistochemical analyses showed that zebrafish expressing mTDP-43 exhibited abnormal increased motor axon branching and NMJ structures with an increase in orphaned presynaptic terminals (Figure 3, A and B) but not postsynaptic structures (Figure 3C). These motor axonal and NMJ phenotypes were partially rescued by incubation with pimozide $(1 \mu \mathrm{M}$, Figure $2 \mathrm{D}$ and 


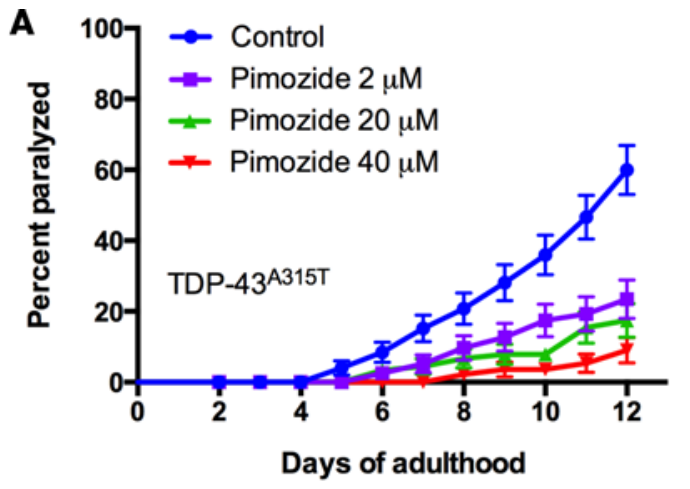

C

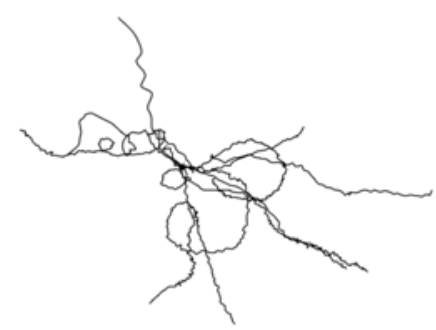

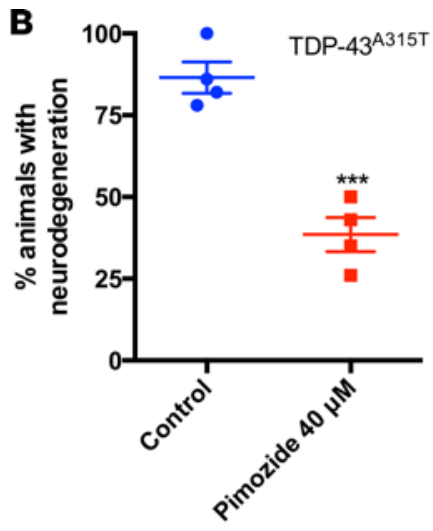

mTDP-43

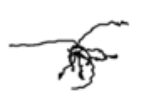

mTDP $-43+1 \mu \mathrm{M}$ pimozide
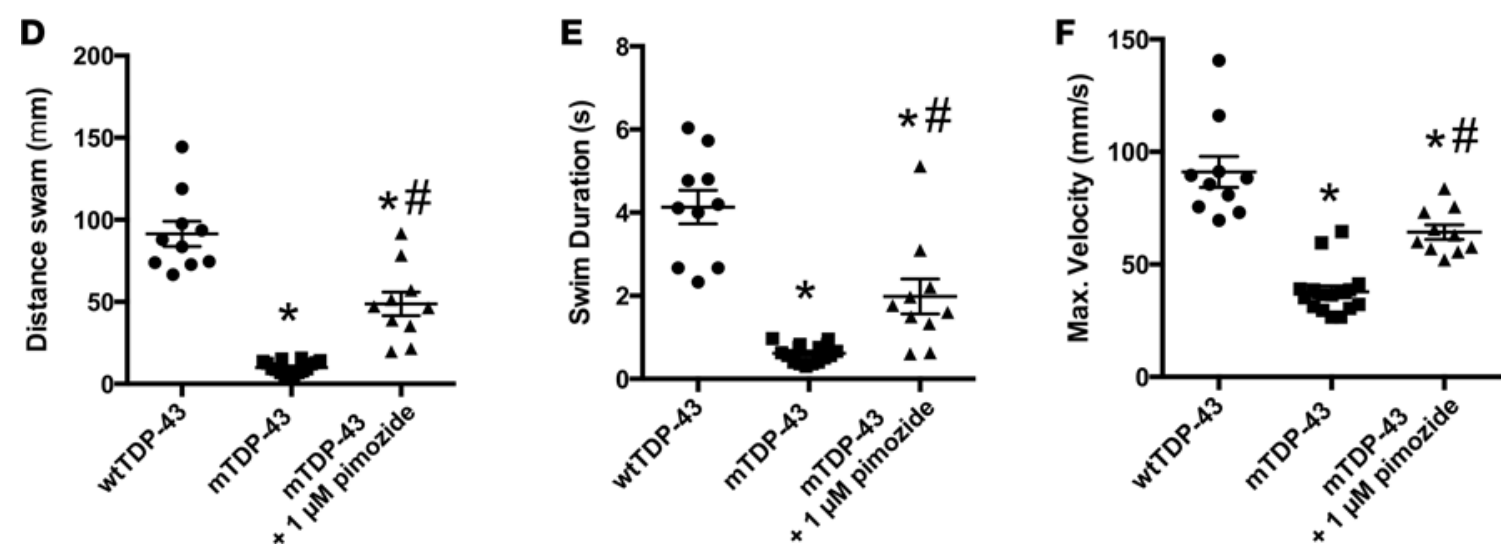

Figure 2. Pimozide reduce motor defects in C. elegans and zebrafish expressing mutant TDP-43. (A) Pimozide reduced mTDP-43-induced paralysis in worms at 3 doses $(2 \mu \mathrm{M}[n=109], 20 \mu \mathrm{M}[n=115]$, and $40 \mu \mathrm{M}[n=111])$ compared with untreated control $(n=125)(P<0.001)$. (B) Pimozide reduced degeneration of motor neuron in mTDP-43 transgenic worms. ${ }^{* *} P<0.001$ ( $n=4$ batches, each batch consisted a quantification of 100 worms). (C) Representative traces of touch-evoked escape response phenotypes in wtTDP-43, mTDP-43, and mTDP-43+pimozide. Application of pimozide (1 $\mu$ M) led to a significant improvement in the distance swam (D), swim duration (E), and maximum swimming velocity (F) of mTDP43 $(n=16)$. Data are represented as mean \pm SEM. Significant difference from wtTD-P43, ${ }^{*} P<0.001$; significantly different from mutant fish, $\# P<0.05$. All data values are given as mean \pm SEM. Significance was determined using one-way ANOVA and Fisher LSD tests for normally distributed and equal variance data; Kruskal-Wallis ANOVA and Dunn's method of comparison were used for nonnormal distributions.

Figure 3, A and B). These results demonstrate that pimozide can significantly reduce the motor neuron phenotypes elicited by expression of mTDP-43 in our genetic models of ALS.

To determine if pimozide is neuroprotective in other models of ALS, we tested it on zebrafish FUS and SOD1 models (26). We found that zebrafish expressing mutant human FUS (FUS ${ }^{\mathrm{R} 521 \mathrm{H}}$, or mFUS; Figure 4, A-C) or SOD1 (SOD1G93A, or mSOD1; Figure 4, D-F) also had impaired swimming, as assessed by their ability to produce an impaired touch-evoked escape response. When treated with pimozide $(1 \mu \mathrm{M}), \mathrm{mFUS}$ and $\mathrm{mSOD} 1 \mathrm{fish}$ showed an improved swimming response, with improved swim duration, distance swam, and maximum swim velocity (Figure 4). Altogether, these data show that overnight treatment with pimozide is neuroprotective in TARBDP, as well as in FUS and SOD1 genetic models of ALS, preventing the phenotype regardless of genotype. 
A

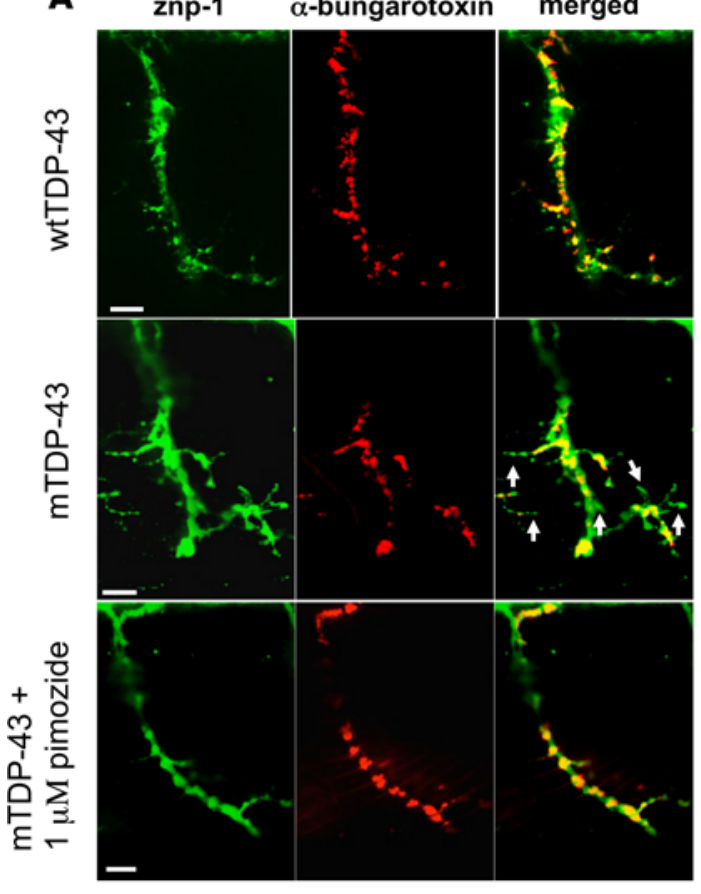

B
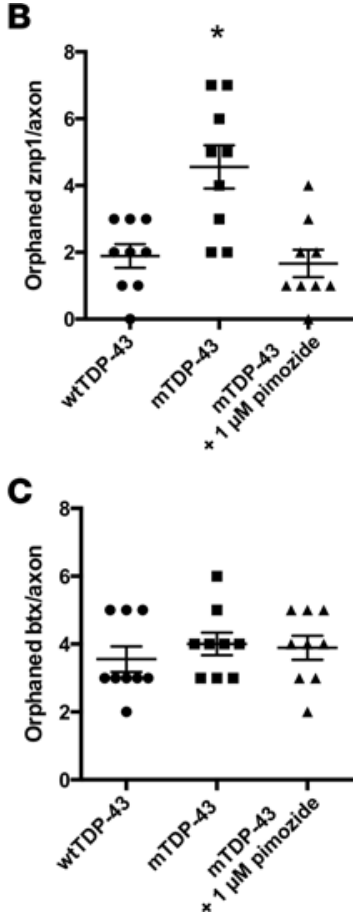

Figure 3. Zebrafish expressing mTDP-43 displayed orphaned presynaptic endings that could be rescued by pimozide. We examined the morphology of the neuromuscular junctions in zebrafish for evidence of mismatched presynaptic and postsynaptic structures. (A) Representative images of 1 ventral root projection double labeled for znp-1 (presynaptic marker) and $\alpha$-bungarotoxin (postsynaptic marker). wtTDP-43 fish showed extensive colocalization of both znp-1 and $\alpha$-bungarotoxin (merged) compared with mTDP-43, where we observed an increase in the number of orphaned znp-1 labeling (B; also see arrows in $\mathbf{A}$ ) but no significant change in the number of orphaned $\alpha$-bungarotoxin labeling (C). These motor axonal anomalies in MTDP-43 were significantly reduced following pimozide treatment (righthand data sets). All data values are given as mean \pm SEM. Significance was determined using one-way ANOVA and Fisher LSD tests for normally distributed and equal variance data; Kruskal-Wallis ANOVA and Dunn's method of comparison were used for nonnormal distributions.

Pimozide acutely restores neuromuscular transmission in C. elegans, zebrafish, and mouse models of ALS

We next sought to assess the effects of pimozide on NMJ transmission in our mTDP-43 models. We assessed the $C$. elegans NMJ following pimozide treatment by measuring the effects of the cholinesterase inhibitor aldicarb in paralyzing mTDP-43 transgenic animals (27). Untreated mTDP-43 worms were strongly sensitive to aldicarb as compared with WT TDP-43 (wtTDP-43) worms (Figure 5A), whereas following treatment with pimozide, mTDP-43 worms showed a significant increase in resistance to aldicarb, suggesting that pimozide stabilized neuromuscular function in animals expressing mTDP-43.

We next tested if pimozide had protective effects at the vertebrate NMJ in our MTDP zebrafish model. Through paired motoneuron-muscle cell recordings, we assessed the fidelity of synaptic NMJ transmission and the strength of muscle endplate currents, as in our previous works $(14,28)$, in mTDP-43 fish treated or not overnight with pimozide. Compared with wtTDP43, the fidelity of NMJ synaptic transmission was greatly reduced in larvae expressing mTDP-43 (Figure 5, B-E, $P<0.05$ ). Following pimozide treatment, we observed a clear stabilization of NMJ efficacy (Figure 5C, $P<0.05$ ) and amplitude (Figure 5D, $P<$ 0.05 ) and a reduced variation in muscle EPC amplitude (Figure 5E, $P<0.05$ ) in treated mTDP-43 fish.

To determine if pimozide has a similar beneficial effect in mammalian models of ALS, we next investigated the effects of acute, 30-minute pimozide treatment on synaptic strength at the NMJ of the $S O D 1^{\mathrm{G} 37 \mathrm{R}}$ mouse, a model characterized by a late-onset hindlimb paralysis (rather than the rapidly progressing phenotype in $S O D 1^{\mathrm{G} 93 \mathrm{~A}}$ mice) that fully recapitulates the ALS phenotype (29). We determined NMJ synaptic strength by performing intracellular recordings at NMJs from the extensor digitorum longus (EDL) muscle (Figure 6, A and B), a fast-twitch muscle essentially composed of fast-fatigable motor units, which are known to be selectively vulnerable in ALS (30-32). We showed that the amplitude of evoked endplate potentials (EPPs) as well as the quantal content (QC) in mutant SOD1 mice were significantly reduced compared with WT mice and pimozide-treated NMJs at both symptomatic (P400) (Figure 6, $\mathrm{C}$ and $\mathrm{D}, P<0.05$ ) and presymptomatic (P160) (Figure 6, G and $\mathrm{H}$ ) stages. At P400, mEPP frequency was significantly reduced in SOD1 NMJs compared with WT mice, while pimozide-treated SOD1 NMJs showed no difference compared with WT control NMJs (Figure 6E). In addition, we observed an increase in miniature endplate potential (mEPP) amplitude in both SOD1 and SOD1treated NMJs compared with WT NMJs (Figure 6F). On the other hand, no differences were found in mEPP frequency between WT and mutant SOD1 mice at P160 in any groups (Figure 6I), as well as in mEPP amplitude (Figure 6J). 
A
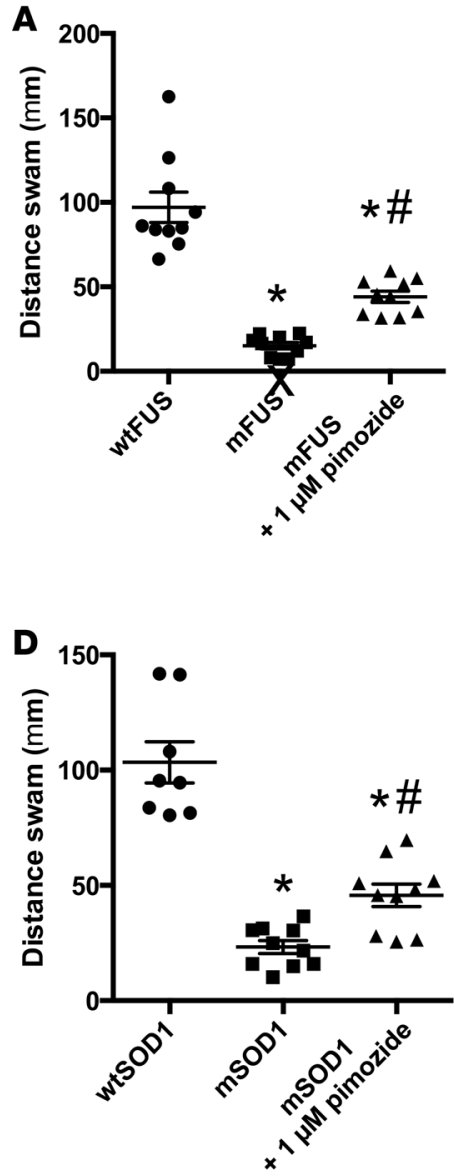
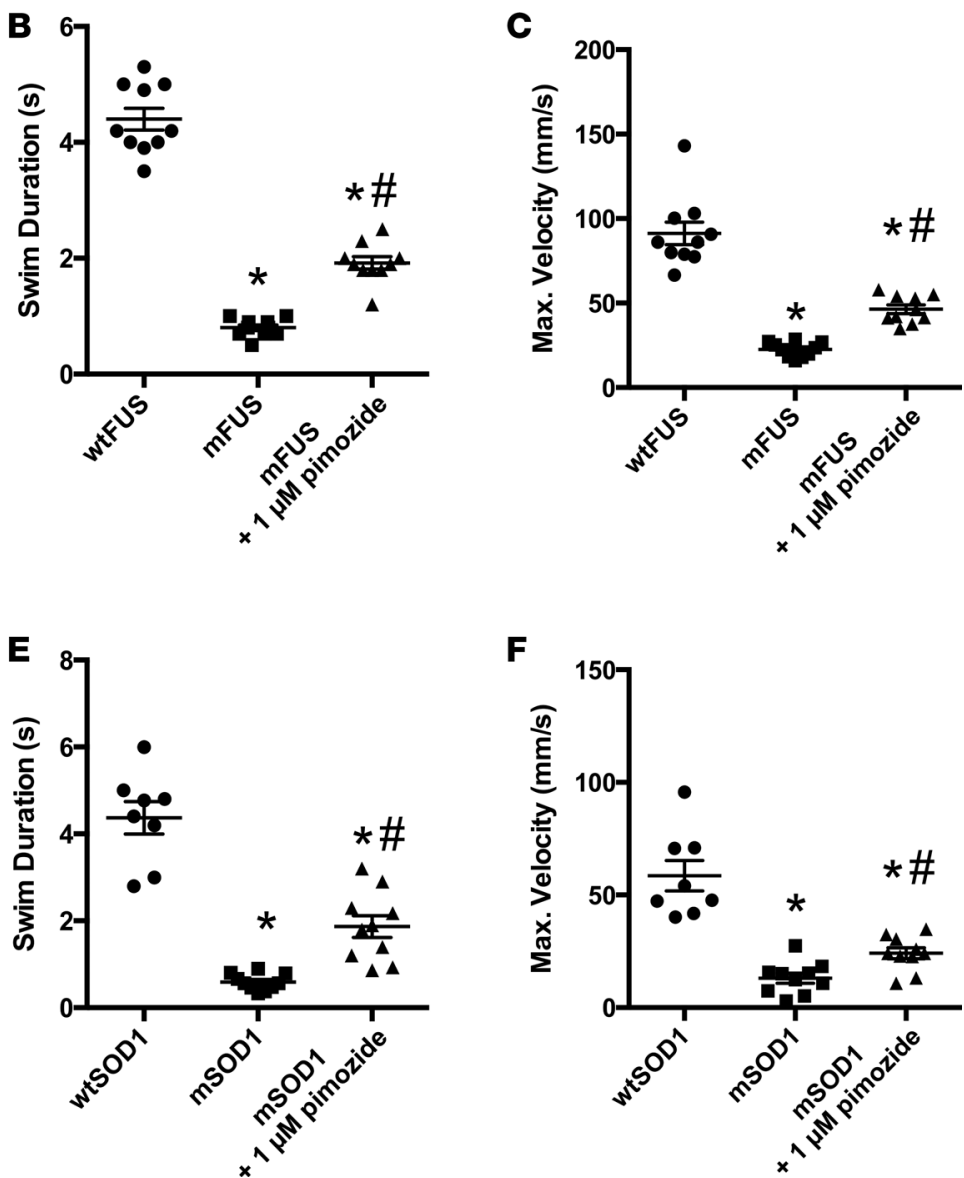

$\mathbf{F}$

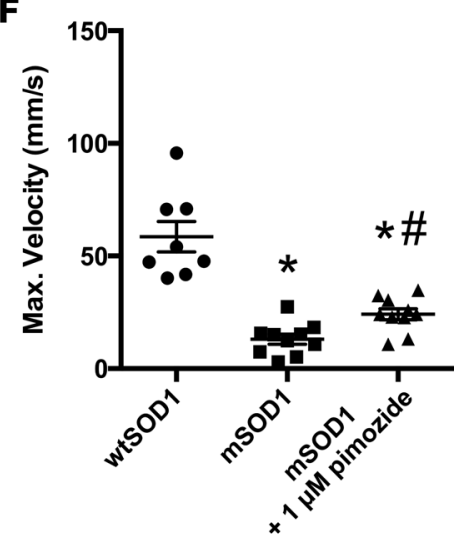

Figure 4. Pimozide reduced motor deficits in zebrafish expressing mutant FUS and SOD1. To extend our observations with TDP-43 models, we tested pimozide on zebrafish expressing other ALS genes, mutant human FUS or SOD1. Application of pimozide (1 $\mu$ M) led to a significant improvement in the distance swam (A), swim duration (B), and maximum swimming (C) velocity of zebrafish expressing mFUS. Treatment with pimozide $(1 \mu \mathrm{M})$ significantly improved the distance swam (D), swim duration (E), and maximum swimming (F) velocity of mSOD1. Significant difference from WT, ${ }^{*} P<0.001$; significantly different from mutant fish, ${ }^{P} P<0.05$. All data values are given as mean \pm SEM. Significance was determined using one-way ANOVA and Fisher LSD tests for normally distributed and equal variance data; Kruskal-Wallis ANOVA and Dunn's method of comparison were used for nonnormal distributions.

In order to confirm that the restoration of NMJ synaptic transmission was caused directly by the effect of the drug, we performed paired electrophysiological recordings following acute bath application of pimozide $(5 \mu \mathrm{M})$ for 30 minutes on a given NMJ previously recorded from. Interestingly, we observed, in all cases, a strong restoration in the amplitude of nerve-evoked response EPPs (Figure 7, A and $\mathrm{E}$ ) and $\mathrm{QC}$ at the presymptomatic stage at $\mathrm{P} 160$, as well as at time of disease onset at P400 (Figure 7, B and F). Pimozide treatment led, in all cases, to an increase in mEPP frequency (Figure 7, C and G) but had no effect on the mEPP amplitude either at P160 or P400 (Figure 7, D and H). Thus, pimozide appeared to enhance NMJ transmission at the SOD1 mouse NMJ.

\section{Pimozide antagonizes T-type Ca ${ }^{2+}$ channels}

While pimozide acts at the NMJ in all of our models, it was designed as a dopaminergic D2 antagonist; however, dopaminergic activity does not contribute to the motor functions we assayed. In considering the possible molecular target of pimozide, we note that many neuroleptics also display off-target effects such as $\mathrm{Ca}^{2+}$ channel blocking activity $(33,34)$. For instance, pimozide has been shown to block low-voltage-activated T-type $\mathrm{Ca}^{2+}$ currents in various cell types (34-36) including human T-type $\mathrm{Ca}^{2+}$ channels expressed heterologously (33). To test if pimozide is acting on T-type $\mathrm{Ca}^{2+}$ channels as a molecular target in our ALS models, we first examined the motility of mTDP-43 C. elegans upon treatment with 3 specific T-type channel antagonists: ML218, NNC-55-0396, and mibefradil. Interestingly, we found that treatment with each of these T-type channel antagonists significantly prevented the 
A

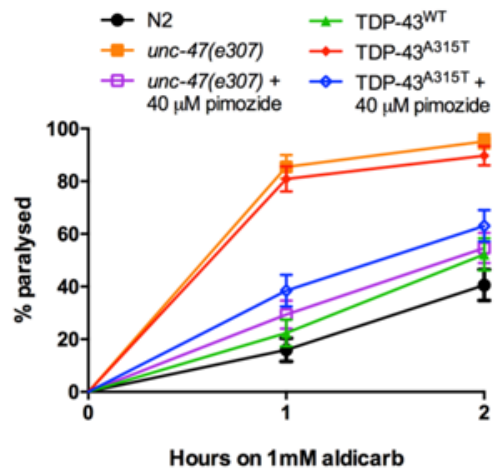

\section{B}
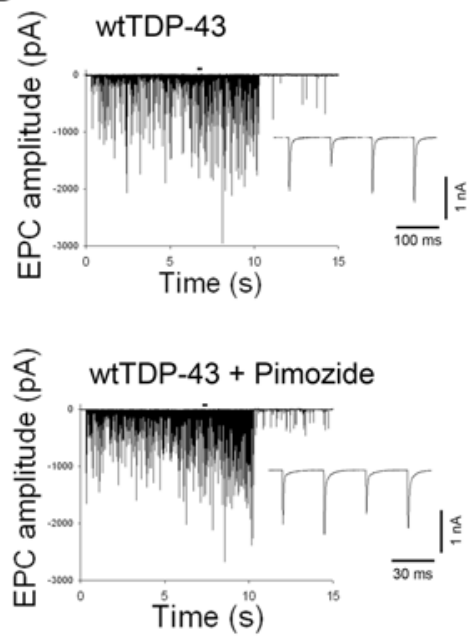

C

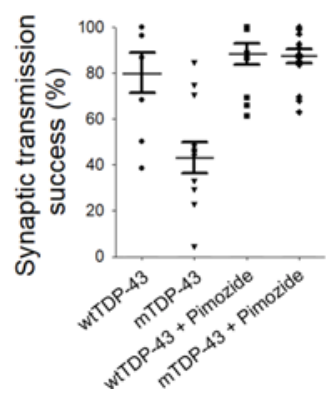

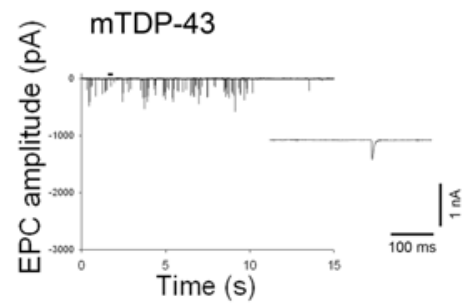

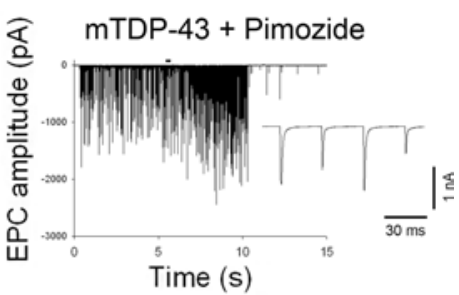

D

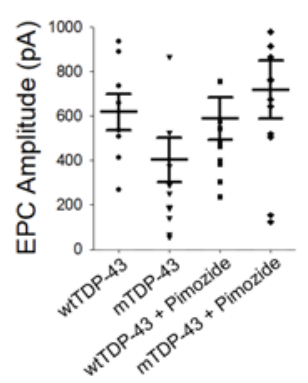

E

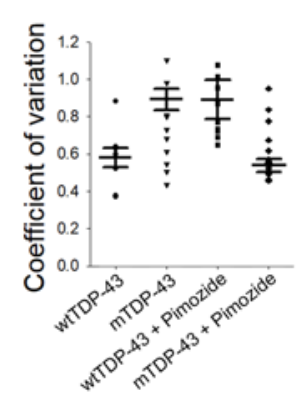

Figure 5. Pimozide restores synaptic transmission in $C$. elegans and zebrafish mutant TDP-43. (A and B) Cholinergic neuronal transmission was measured by determining the onset of paralysis induced by the cholinesterase inhibitor aldicarb. mTDP-43 transgenics and unc-47 (e307) mutants were hypersensitive to aldicarb-induced paralysis compared with either wtTDP-43 transgenics or N2 worms $(P<0.001$ for unc-47 $[n=61]$ or mTDP-43 [ $n$ = 67] compared with N2 [ $n=69]$ or wtTDP-43 worms [ $n$ = 66]). Pimozide reduced the aldicarb-induced paralysis in mTDP-43 transgenics $(n=63)$ as well as unc-47 (e307) mutants $(n=74)(P<0.001)$. To assess synaptic transmission across the NMJ in zebrafish models of ALS, paired recordings (primary motoneuron-muscle [MN-muscle]) were preformed. (B) Example traces of EPCs in fast-twitch muscles in wtTDP-43 and mutTDP-43 fish with and without pimozide were recorded following a 10 -second train of depolarizing (action potential generating); current steps delivered at $30 \mathrm{~Hz}$. Pimozide treatment was found to recover the fidelity of synaptic transmission (C), the EPC amplitude (D) and the coefficient of variation (E) in mTDP43 fish $(n=10)$. Data are represented as mean \pm SEM. Significance was determined using one-way ANOVA and Fisher LSD tests for normally distributed and equal variance data, Kruskal-Wallis ANOVA and Dunn's method of comparison were used for nonnormal distributions.

paralysis phenotype in mTDP-43 worms (Figure 8A, $P<0.001$ ). To confirm that pimozide can directly antagonize T-type $\mathrm{Ca}^{2+}$ channels, we recorded low- and high-voltage-activated $\mathrm{Ca}^{2+}$ currents in zebrafish motoneurons in the presence or absence of pimozide. We found that pimozide selectively blocked the low- (and not the high-) voltage-activated $\mathrm{Ca}^{2+}$ current that is mediated by T-type $\mathrm{Ca}^{2+}$ channels in zebrafish (Figure 8B). Together, our data suggest that pimozide acts on T-type $\mathrm{Ca}^{2+}$ channels in our ALS models to enhance NMJ activity.

\section{Pimozide therapeutic effects assessed in human subjects with ALS}

Our findings above indicate that pimozide can ameliorate locomotor function acutely by stabilizing synaptic transmission defects at the NMJ caused by ALS mutations in a variety of genetic models. Very limited clinical information on the use of pimozide in subjects with ALS is available (37). We therefore conducted a pilot RCT testing pimozide versus placebo in subjects with ALS to identify the maximum dose that was safe and tolerable, assess for acute target engagement at the NMJ, and investigate for preliminary evidence of an effect of pimozide on clinical and electrophysiological outcome measures. 
A

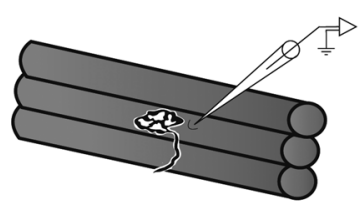

B

WT

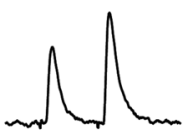

SOD1 - Ctrl

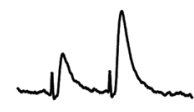

SOD1 - Pim

\section{Symptomatic - P400}
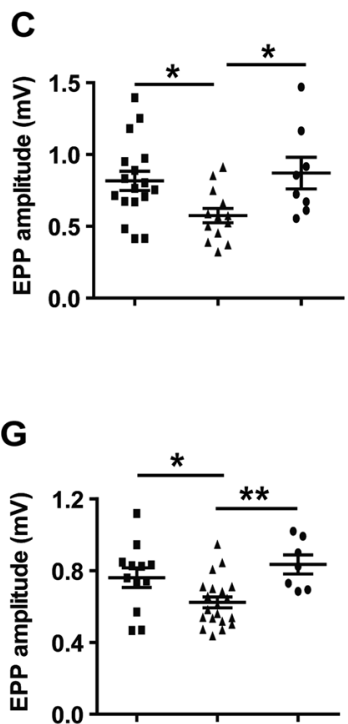

D

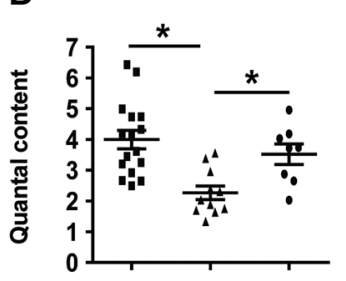

Presymptomatic - P160

H

E

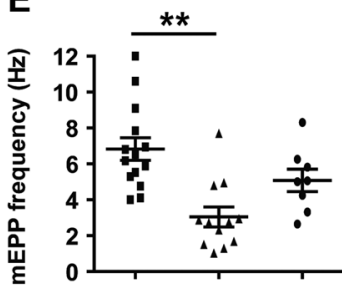

I

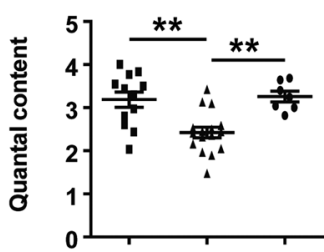

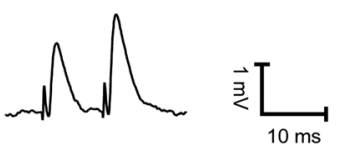

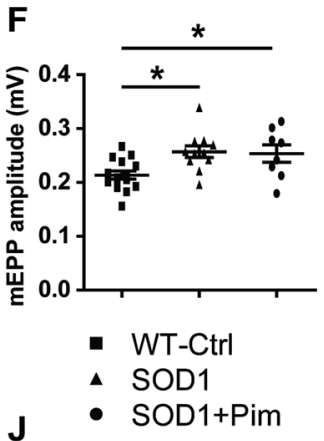

J

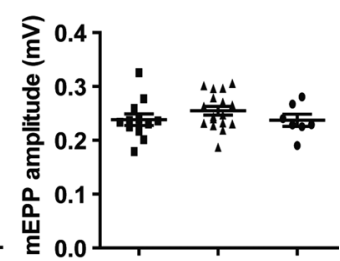

Figure 6. Pimozide rescues synaptic strength deficits in the SOD1 ${ }^{\mathrm{C37R}}$ EDL at disease onset (P400) and at the presymptomatic stage (P160). (A) Schema of the electrophysiology experiment. Muscles were impaled at $50 \mu \mathrm{m}$ from an adjacent NMJ to perform intracellular recordings of EPP amplitude. (B) Example traces of nerve-evoked EPPs following a paired-pulse facilitation protocol elicited at $0.2 \mathrm{~Hz}$, for WT (left), nontreated SOD1 (middle, SOD1-Ctrl), and pimozide-treated NMJs (right, SOD1-Pim). Histograms show the mean \pm SEM of EPP amplitude at P400 (C) and P160 (C), quantal content (D and H), mEPP frequency (E and I), and mEPP amplitude ( $F$ and J) of the WT, SOD1, and SOD1-Pim NMJs from WT and SOD1C37R mice. Quantal content was obtained by dividing the mean EPP of the first EPPs, including failures, divided by the mean mEPP amplitude. ${ }^{*} P<0.05,{ }^{*} P<0.01$. The sample numbers were as follows: (for P400) WT, $n=16$; SOD1, $n=12$; SOD1-Pim, $n=8$; (for P160) WT, $n=13$; SOD1, $n=17$; SOD1-Pim, $n=8$. All data values are given as mean \pm SEM. Significance was determined using one-way ANOVA and Fisher LSD tests for normally distributed and equal variance data; Kruskal-Wallis ANOVA and Dunn's method of comparison were used for nonnormal distributions.

We chose a 6-week treatment period, as this should be just long enough to assess the safety and tolerability profile in ALS subjects and to observe for acute effects of pimozide on abnormal decremental responses on RNS. Twenty-five subjects were randomized, with 1 active treatment patient discontinuing the study due to death, as summarized in the CONSORT flow diagram (Figure 9).

Safety and tolerability. To examine safety and tolerability, pimozide was first uptitrated in 2 groups to doses of $10 \mathrm{mg} /$ day (the maximal dose typically used in patients for approved clinical indications) and 6 $\mathrm{mg} /$ day, respectively, but it was poorly tolerated. The protocol was amended to reduce the doses to $4 \mathrm{mg} /$ day and $2 \mathrm{mg} /$ day as subjects tolerated those doses in the uptitration phase and following reductions from higher doses. As a result of this change in protocol, we have defined low dose as $2 \mathrm{mg} /$ day and high dose as $4 \mathrm{mg} /$ day or higher. At the $4 \mathrm{mg}$ /day high dose and $2 \mathrm{mg}$ /day low dose, there was an increased frequency of reported generalized weakness and akathisia $(62.5 \%$ at $4 \mathrm{mg} /$ day high dose, $25 \%$ at $2 \mathrm{mg}$ /day low dose, $0 \%$ with placebo) of mild to moderate severity as compared with the placebo group.

Target engagement. Due to the expected dysfunction of the NMJ, we compared changes in decremental responses on repetitive nerve-muscle stimulation between the randomization visit and end-of-treatment visit. We assessed CMAP decrement in the median, ulnar and accessory nerves by recording CMAPs of $\mathrm{APB}, \mathrm{ADM}$ and trapezius, respectively, and examined the changes in percent decremental response to RNS between the randomization visit and end-of-treatment visit, as the APB is one of the first muscles to show weakening in ALS. While we found no significant changes in left APB, bilateral ADM, or trapezius muscles (Figure 10), we observed a significant difference in change in percent decremental response of righthand 
Presymptomatic - P160

A

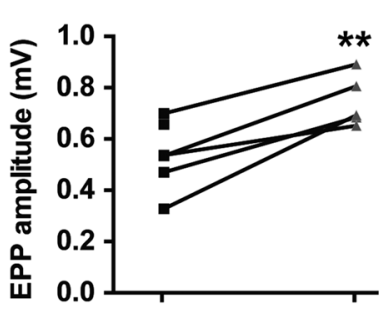

B

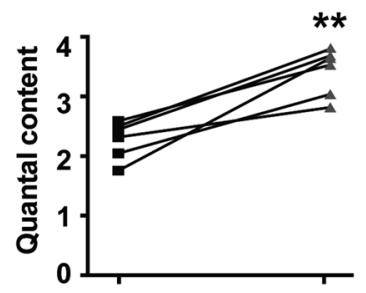

C

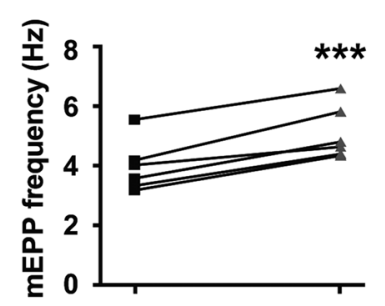

D

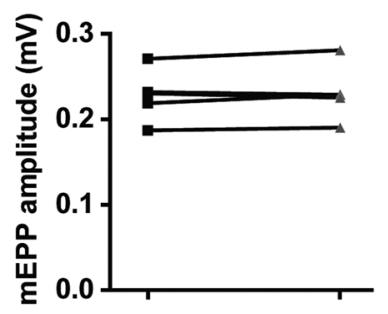

Symptomatic - P400

G

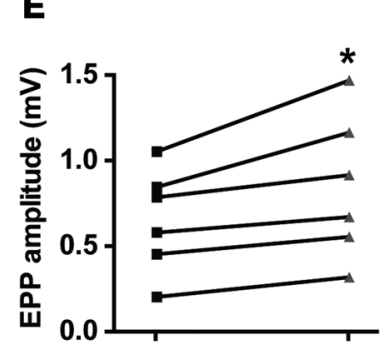

$\mathbf{F}$

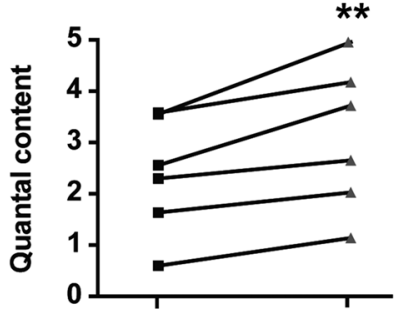

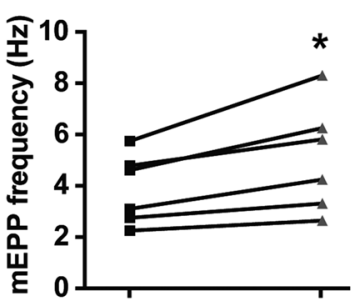

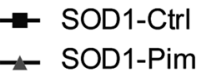

H

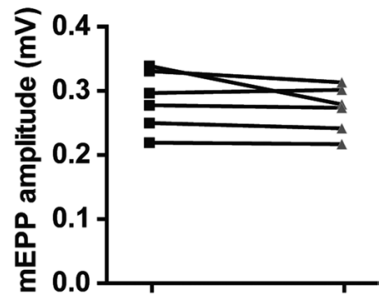

Figure 7. Persistent synaptic strength alterations were rescued by Pimozide in the SOD1C37R EDL. Paired experimental results showing synaptic strength before and after a 30- to 45 -min bath application of pimozide $5 \mu \mathrm{M}$, on the same neuromuscular junction. Pimozide-enhancing effect on synaptic transmission was clearly demonstrated at the presymptomatic stage as well as disease onset on evoked endplate potential (EPP) amplitude (A and E), quantal content (B and $\mathbf{F}$ ), miniature endplate potential frequency (mEPP frequency) (C and $\mathbf{G}$ ), and mEPP amplitude ( $\mathbf{D}$ and $\mathbf{H})$ before (square) and after pimozide bath application in SOD1 neuromuscular junctions (triangle). Quantal content was obtained by dividing the mean EPP of the first EPPs, including failures, by the mean mEPP amplitude. ${ }^{*} P<0.05,{ }^{* *} P<0.01,{ }^{* *} P<0.001$. All data values are given as mean \pm SEM. Significance was determined using Students 2 -tailed $t$ test.

APB between those in the placebo group versus those who received either high dose (subjects each receiving 10,6 , or $4 \mathrm{mg} /$ day) or low dose ( $2 \mathrm{mg} /$ day) of pimozide (Figure $11, \mathrm{~A}$ and $\mathrm{B}, P<0.05$ ), suggesting protection against worsening of decremental responses in the right APB muscle. These results suggest a stabilization of NMJ function in the only muscle assessed that showed a change in decrement over the study interval.

Clinical outcome measures. As part of our clinical assessment, we performed the revised ALS Functional Rating Scale (ALSFRS-R), which includes measures of mobility and respiration; slow vital capacity (SVC), a measure of respiration; and motor power Medical Research Council (MRC) sum score. All analyses were performed using intention-to-treat, with the last observation carried forward for dropouts, including early discontinuations $(n=3)$. We examined the change in ALSFRS-R between randomization and endof-treatment visits and found no significant difference in change between placebo, low-dose, and high-dose groups (Figure 11C). Similarly, change in SVC between randomization and end-of-treatment visits was calculated and revealed no significant difference between placebo, low-dose, and high-dose groups (Figure 11D). Regression analyses examining for a relationship between dose actually received and change in ALSFRS-R and SVC did not reveal any significant associations. While analysis of motor power (MRC sum score) revealed a significant progression of weakness in patients receiving placebo, in contrast, a lack of progression of weakness was observed in patients on low and high pimozide doses (Figure $11 \mathrm{E}, P<0.05$ ).

Altogether, these data suggest that the maximal safe and tolerable dose of pimozide in subjects with ALS is $4 \mathrm{mg} /$ day, and there is preliminary evidence of target engagement at the NMJ. The brief period of treatment with pimozide did not impact clinical or respiratory function, neither of which significantly declined in the placebo group. Lack of deterioration of the motor power as measured by the MRC sum score in the low- and high-dose treatment groups may reflect early clinical benefit, but the imbalance of baseline characteristics with subjects with more rapidly progressing and more advanced ALS in the placebo group preclude any conclusions of efficacy. Future studies should include the MRC sum score as an outcome measure to investigate further. 


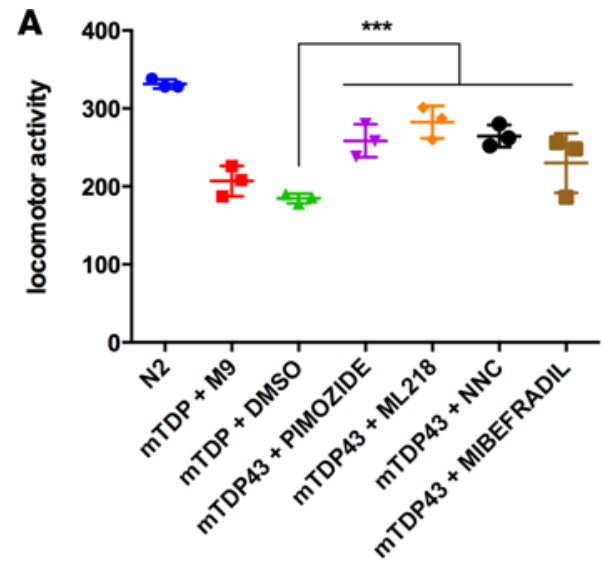

B

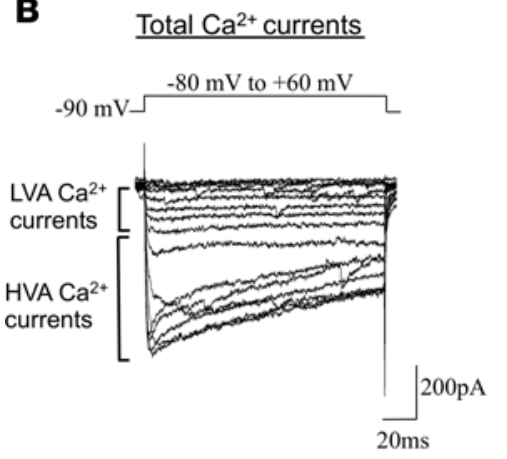

Isolated LVA Ca ${ }^{2+}$ currents
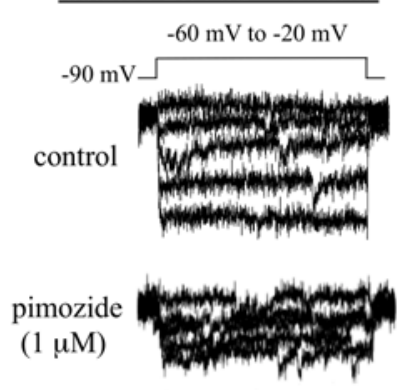

$\frac{5}{20 \mathrm{~ms}} \mathrm{pA}$

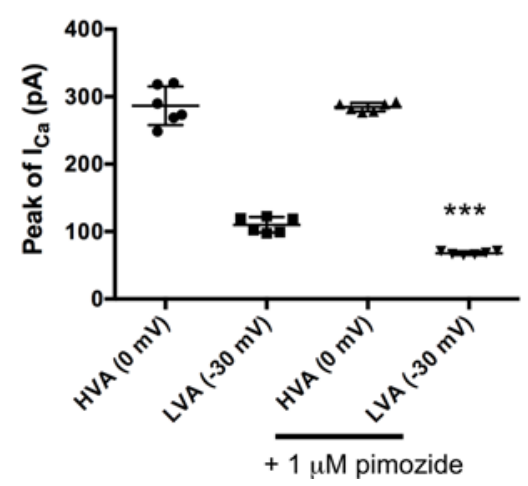

Figure 8. Pimozide antagonizes T-type $\mathrm{Ca}^{2+}$ channels. (A) Three T-type $\mathrm{Ca}^{2+}$ channel antagonists (ML218, NNC, and miberfradil) at $20 \mu \mathrm{M}$ improved motor phenotype in mTDP43 C. elegans ( $n=3$ batches, each batch consisted a quantification of 30 worms). (B) Total voltage-gated calcium currents mediated by high-voltage-activated (HVA) and low-voltageactivated $\mathrm{Ca}^{2+}$ channels (LVA) were isolated by patch-clamp recording in vivo in $\mathrm{mTDP}-43$ zebrafish motoneurons using a voltage-clamp protocol (depolarizing from $-80 \mathrm{mV}$ to +60 $\mathrm{mV}$ ) (left panel). Pimozide antagonizes LVA Ca ${ }^{2+}$ currents mediated by T-type $\mathrm{Ca}^{2+}$ channels (isolated by holding at $-30 \mathrm{mV}$ ) in zebrafish motoneurons $(n=6)$. Representative recording traces of isolated LVA $\mathrm{Ca}^{2+}$ currents with or without pimozide are presented in the right panel, and the effects of pimozide on HVA and LVA Ca ${ }^{2+}$ currents are illustrated in the bar graph. ${ }^{* *} P<0.001$. All data values are given as mean \pm SEM. Significance was determined using one-way ANOVA and Fisher LSD tests for normally distributed and equal variance data; Kruskal-Wallis ANOVA and Dunn's method of comparison were used for nonnormal distributions.

\section{Discussion}

Using our in vivo models, we performed a large-scale chemical screen and identified 13 neuroleptics that acutely stabilized motor function in $C$. elegans, and most of these were active in zebrafish expressing mutant TARDBP. The lead compound, pimozide, was found to maintain NMJ structure, as well as transmission, upon repetitive stimulation in mTDP-43 zebrafish. Pimozide was also effective in enhancing synaptic transmission at NMJs in SOD1 $1^{\mathrm{G} 37 \mathrm{R}}$ mice. In a small phase II RCT of pimozide over a 6-week period, we demonstrated that the maximal safe and tolerable dose of pimozide in subjects with ALS is $4 \mathrm{mg}$ / day, obtained preliminary evidence of target engagement at the NMJ, and we conclude that a larger number of subjects with a longer treatment interval is now required to assess for clinical efficacy.

In our study, we combined the use of several model systems (C. elegans, zebrafish, and mice) and have capitalized on the strength of each organism to identify small molecule therapeutics for ALS. We identified the lead compound pimozide to ameliorate motor function in all the model organisms and translated the findings into a clinical trial for ALS. Our findings demonstrate that simple animal models can be valuable in the preclinical pipeline to bridge the gap between in vitro assays and more costly screens in mammals. More importantly, the effect of pimozide in our $C$. elegans and zebrafish models of ALS and humans suggests not only that simple animal models are useful in identifying compounds that hold promise for the treatment of ALS, but they may be accurate predictors of clinical trial outcomes.

Pimozide is an FDA-approved neuroleptic used to treat chronic psychosis, Tourette syndrome, and resistant tics (38). It has primarily dopamine D2 receptor-blocking activity (39), but the motor behaviors we screened are independent of dopamine action. Several studies have reported other, off-target pharmacological properties of pimozide, such as antagonism of T-type $\mathrm{Ca}^{2+}$ channels $(33,36)$, inhibition of STAT5 (40) and STAT3 (41), and autophagy enhancement (42). Less-selective drugs may in fact be better, since they could regulate several cellular targets. We observed an antagonism of T-type $\mathrm{Ca}^{2+}$ channels in TDP worms and fish, as reported previously for human channels, and found that pimozide preserved NMJ transmission in zebrafish and mouse models of ALS. Similarly, we previously showed that other calcium channel drugs (FPL 64176 and Bay K 8644) suppressed the NMJ phenotype in mutant TDP-43 ALS zebrafish (14). Through facilitation 


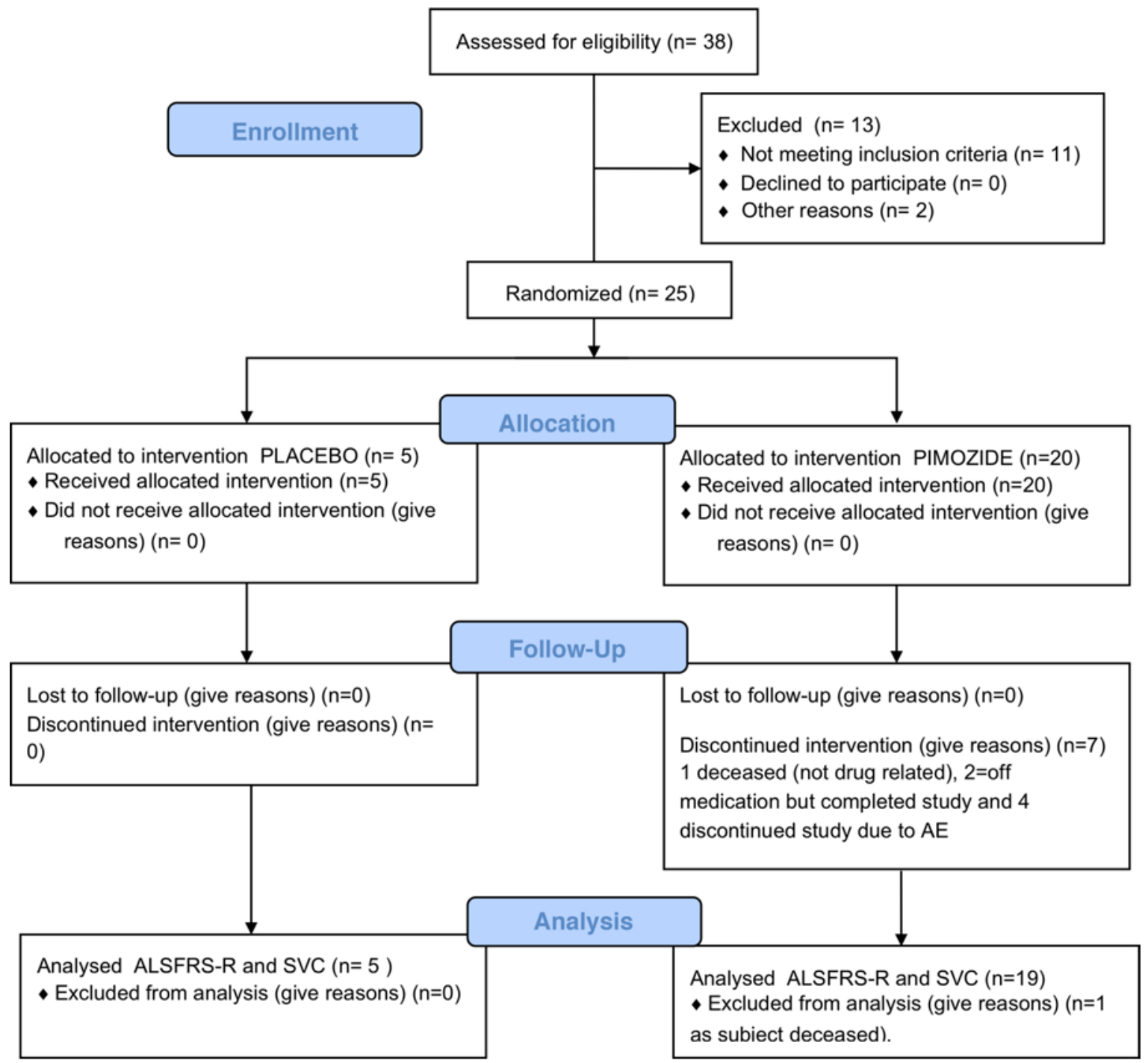

Figure 9. A CONSORT flow diagram of pimozide phase Ilb trial for ALS. Twenty-five subjects were randomized and treated with placebo or pimozide for 6 weeks.

of NMJ transmission, as we observed in zebrafish and mice, pimozide may, thus, act to enhance motor unit function early in the disease course of ALS. The preliminary evidence of target engagement of pimozide at the NMJ in the RCT we report provides data to support a comparable mechanism in human subjects with ALS.

To our knowledge, we report the first pilot randomized double-blinded placebo-controlled RCT of pimozide in subjects with ALS. A previous RCT of selegiline, vitamin E, and pimozide at $1 \mathrm{mg}$ per day was performed in 44 ALS subjects but was unblinded. The investigators concluded that there was a decrease in the rate of change in the Norris Scale in the pimozide group compared with the other groups (37). Our dose uptitration schedule allowed assessment of dose tolerance and demonstrated that doses above $4 \mathrm{mg} /$ day were poorly tolerated, providing a dose ceiling for future studies. We favor a maximum dose of $4 \mathrm{mg} /$ day due to the relatively high proportion (63\%) of subjects reporting mild or moderate akathisia at this dose, which requires study with more chronic administration.

The efficacy and RNS outcomes of this study need to be considered in the light of the inherent limitations of a small sample size with imbalances between groups at baseline. For example, the mean ALSFRS-R, SVC, and MRC sum score of the placebo group at randomization were lower than the 2 active treatment groups, indicating that the placebo group may have had more weakness, lower vital capacity, and reduced function at the time of randomization, consistent with more advanced ALS. Concordantly, the placebo group had abnormal decremental responses in right APB and trapezius muscles at randomization, whereas pimozide groups did not. It is likely that the subjects in the placebo group were progressing faster prior to 
A

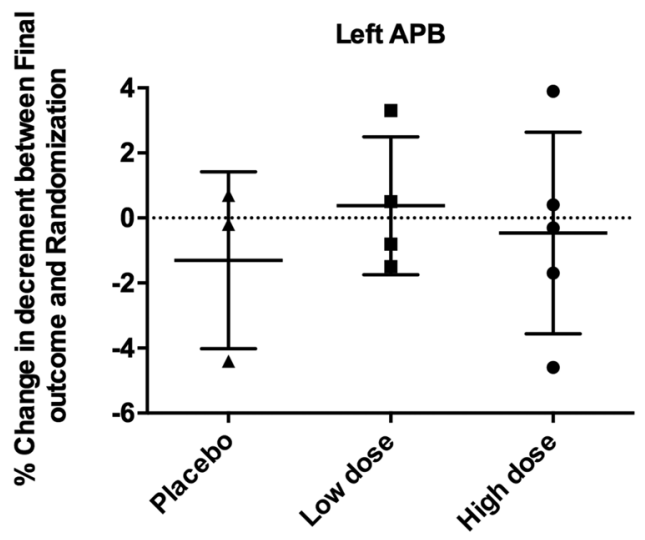

C

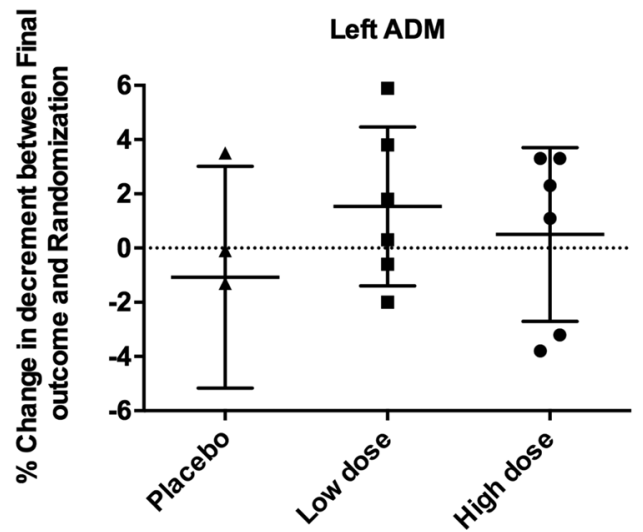

B

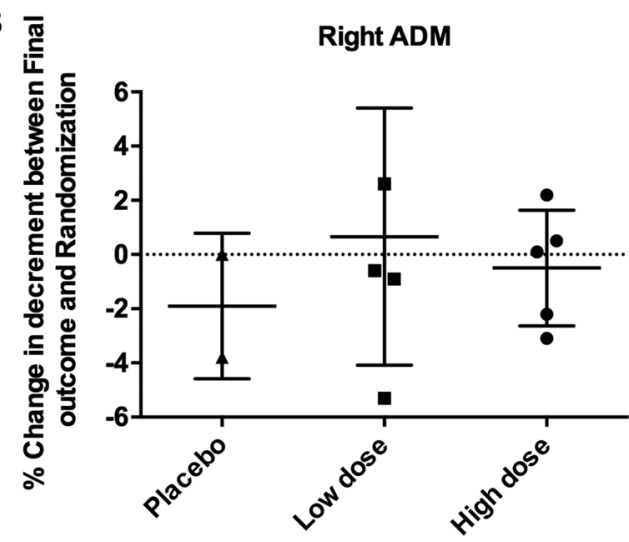

D

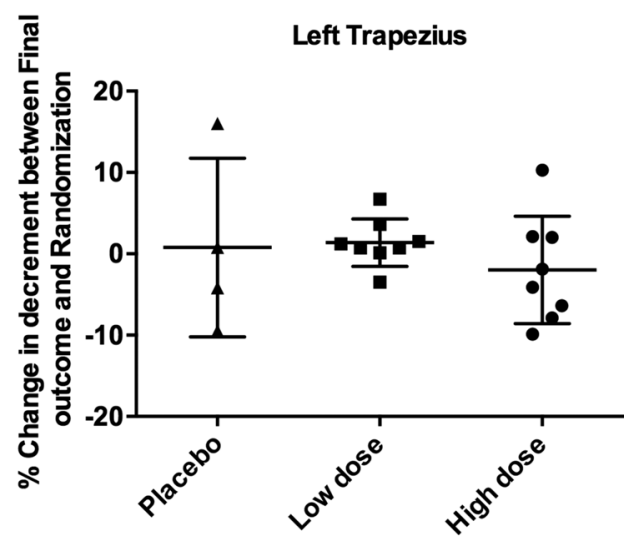

Figure 10. No change in compound motor action potential (CMAP) decrement in left abductor pollicis brevis (APB), right abductor digit minimi (ADM), left ADM, and left trapezius in ALS patients following pimozide treatment. We tested for target engagement in several muscles of patients in which a decrement was not observed during the pimozide treatment period. CMAP recordings of left APB (A), right ADM (B), left ADM (C), and left trapezius (D) muscles were examined for changes in percent decremental response to repetitive nerve stimulation between the randomization visit and end-of-treatment visit. Pimozide did not significantly improve the percentage change in decremental response in CAMP recordings of these muscles. Primary outcome measure analyses were performed using nonparametric analyses including Wilcoxon rank-sum test for change scores between end-of-treatment and randomization visits and Kruskal-Wallis $\mathrm{H}$ test for comparison between multiple groups with correction for repeated measures. Additional linear mixed model regression analysis was performed for analysis of decremental response change from end of treatment based upon actual dose of pimozide.

randomization compared with the 2 treatment groups, as the time to randomization from symptom onset was similar; thus, further study in human subjects is required before conclusions about efficacy can be made.

Consistent with results from our animal models, previous studies have demonstrated abnormal decremental responses in many patients with ALS, indicating abnormal NMJ transmission and correlation of severity of decrement with degree of weakness. This RCT demonstrated preservation of decremental responses in right APB in the treatment groups but worsening in the placebo group, suggesting target engagement of pimozide in the form of protection against worsening of impaired NMJ transmission. It is possible that this was only found in right $\mathrm{APB}$, as that was the only muscle where the placebo group demonstrated worsening (with most patients being right-handed) from a decrement of $-12.5 \%$ to $-24.2 \%$, whereas the other hand muscles (left APB, bilateral ADM) did not show a worsening of decrement from randomization to end-of-treatment visits. These differences are expected due to the well-described split-hand syndrome (43) in ALS, as well as the predominance of ALS signs and symptoms in the right hand over the left hand due the effects of handedness of location of weakness onset in ALS (44). There was no significant worsening of decrement in any treatment group for any muscle in this short trial. These RNS findings and preservation of right APB mean MRC sum score change during the study may represent a sub-clinical electrophysiological effect of pimozide upon the NMJ apparent prior to clinically measurable weakness. The question of whether such electrophysiological effects could result in preservation of motor power over a longer study interval requires further investigation, as will the need to assess this measure as a biomarker of change in motor power and function. 
A

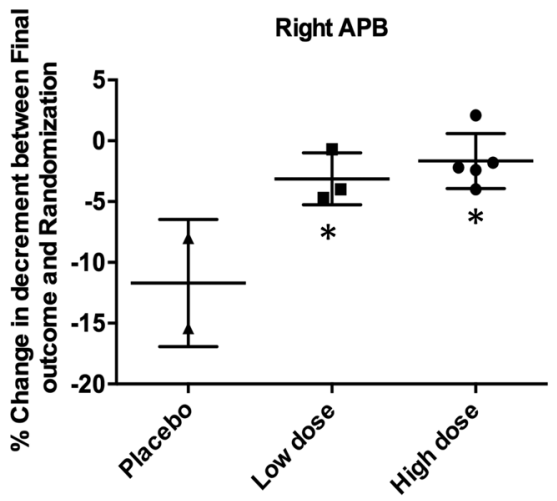

B
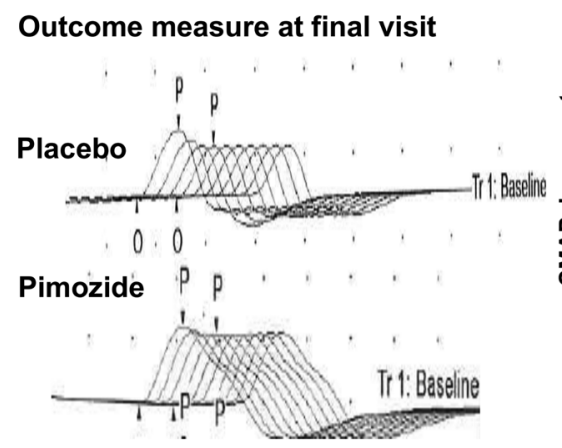

Right APB
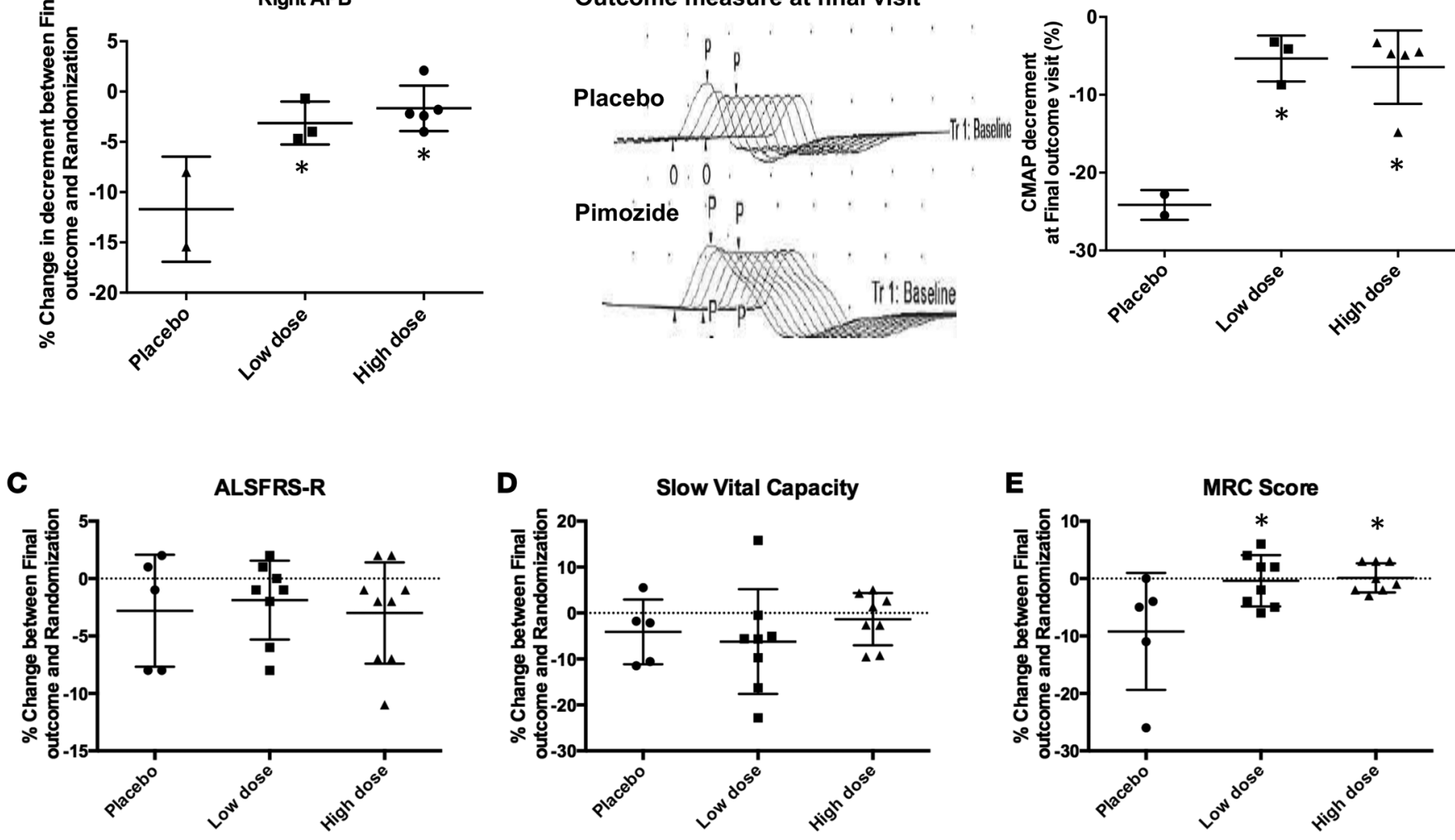

Figure 11. Pimozide improves CMAP decrement in right APB and MRC sum score in ALS patients. Compound motor action potential (CMAP) recordings of right APB (A) were examined for changes in percent decremental response to repetitive nerve stimulation (RNS) between the randomization visit and end-of-treatment visit. Pimozide significantly improved the percentage change in decremental response in CAMP recordings of right APB. Placebo, $n=2$; low dose, $n=3$; high dose, $n$ = 5. (B) Examples of CAMP recordings measured at the right APB at the end of placebo or pimozide treatment visits (left panel). Pimozide treatment was found to improve CAMP decrement recorded at the right APB (right panel). (C-E) Revised ALS Functional Rating Score (ALSFRS-R), slow vital capacity (SVC), and MRC sum score were assessed in patients treated with placebo, low dose $(2 \mathrm{mg} /$ day), or high dose $(4 \mathrm{mg} /$ day or more) of pimozide. The bar graphs represent the change in ALSFRS-R (C), SVC (D), and MRC sum score (E) between randomization and end of treatment visits. ${ }^{*} P<0.05$. Placebo, $n=5$; low dose, $n=8$; high dose, $n=8$. Primary outcome measure analyses were performed using nonparametric analyses including Wilcoxon rank-sum test for change scores between end-of-treatment and randomization visits and Kruskal-Wallis $\mathrm{H}$ test for comparison between multiple groups with correction for repeated measures. Additional linear mixed model regression analysis was performed for analysis of decremental response change from end of treatment based upon actual dose of pimozide.

In summary, we showed that simple genetic models are useful in identifying compounds that hold promise for the treatment of ALS, such as neuroleptics. We found that pimozide is safe and tolerable at doses up to $4 \mathrm{mg}$ /day and demonstrated preliminary evidence of target engagement at the NMJ. It has been proposed that prolonged use of antipsychotic medications may protect against ALS (45) and a recent report has shown a genetic link between ALS and schizophrenia, supporting the idea that neuroleptics may be effective in treating ALS (46). The next RCT of pimozide in 100 subjects with ALS began recruitment in November 2017 (ClinicalTrials.gov; NCT03272503) and will further examine pimozide's effects on safety, tolerability, clinical outcome measures, and RNS in ALS. Further investigation is required to determine if pimozide, or any of the other dozen neuroleptics we have identified, corrects impaired NMJ transmission in subjects with ALS and whether decline of clinical measures can be slowed with more prolonged treatment.

\section{Methods}

\section{C.elegans studies}

Genetic models. Standard methods of culturing and handling C. elegans were followed (22). Worms were maintained on standard nematode growth medium (NGM) plates streaked with OP50 E. coli. Strains and mutants used in this study include: N2, xqIs132[unc-47::TDP-43-WT;unc-119(+)], xqIs133[unc47::TDP-43[A315T];unc-119(+)]. All transgenic strains were previously described (22) and were obtained 
from the C. elegans Genetics Center (University of Minnesota, Minneapolis, Minnesota, USA).

Drug screen assays. The LOPAC1280 library (MilliporeSigma), composed of 1,280 bioactive compounds; the Spectrum library (MicroSource Discovery), containing 2,000 compounds; the Biomol, containing 500 compounds; and the Prestwick library, containing 1,120 compounds, were selected for the worm-based motility screenings. From these partly overlapping libraries, a total of 3,750 compounds were screened. Aliquots of the chemicals ( $1 \mathrm{mM}$ in DMSO) were transferred with a Biomek FX (Beckman) from the mother plates into 96-well culture plates to generate diluted stock plates (20 $\mu \mathrm{M}$ final concentration). Candidate hit compounds from the high-throughput screen were purchased from MilliporeSigma for further testing.

Age-synchronized young adult TDP-43 transgenic worms were distributed in 96-wells plate in M9 buffer (20 $\mu 1$ per well, 20-30 worms per well), containing DMSO or test compounds and incubated for up to 12 hours at $20^{\circ} \mathrm{C}$ on a shaker. Motility was assessed manually by microscopy after 6 hours. Experiments were done in triplicate. Drugs were considered to be active when at least $80 \%$ of mutant worms displayed an improved motility phenotype. All putative positively acting compounds were retested, and comparable results were obtained in each case. Positive-hit compounds were then tested on solid media at different concentrations $(2 \mu \mathrm{M}, 20 \mu \mathrm{M}$, and $40 \mu \mathrm{M})$. Briefly, worms expressing TDP-43 adult day 1 (20-30 worms) of adulthood animals were picked and transferred to NGM plates and scored daily for movement over 12 days. Animals were counted as paralyzed if they failed to move upon prodding with a worm pick as described previously (22). Worms were scored as dead if they failed to move their head after being prodded on the nose and showed no pharyngeal pumping. All experiments were conducted at $20^{\circ} \mathrm{C}$. T-type $\mathrm{Ca}^{2+}$ channel antagonists ML218, NNC-55-0396, and mibefradil were purchased from Tocris Bioscience and tested at $20 \mu \mathrm{M}$.

Neurodegeneration assays. For scoring of neuronal processes for gaps or breakage, TDP-43 transgenic animals were selected at day 9 of adulthood for visualization of motor neuron processes in vivo. Animals treated with or without compounds were immobilized in $\mathrm{M} 9$ with $5 \mathrm{mM}$ levamisole and mounted on slides with $2 \%$ agarose pads. GFP was visualized at $505 \mathrm{~nm}$ using a Zeiss Axio Imager M2 microscope. The software used was Zen Pro 2012. One hundred worms were scored per condition.

Synaptic transmission test. Young adults (20-30 worms) were transferred to NGM with $1 \mathrm{mM}$ aldicarb plates. Worms were scored every 30 minutes for 2 hours and counted paralyzed if they failed to move after being prodded on the nose. Experiments were conducted at $20^{\circ} \mathrm{C}$ and done in triplicates.

\section{Zebrafish studies}

Genetic models. Zebrafish (Danio rerio) embryos of the TL line (obtained from the Zebrafish International Resource Center, University of Oregon, Eugene, Oregon, USA) were raised at $28.5^{\circ} \mathrm{C}$, and they were collected and staged using standard methods (47). Human FUS WT and mutant (R521H), human TDP-43 WT and mutant (G348C), and human SOD1 WT and mutant (G93A) mRNAs were transcribed from NotI-linearized pCS2+ using SP6 polymerase with the mMESSAGE Machine Kit (Ambion). This was followed by a phenol-chloroform extraction and isopropanol precipitation, and diluted in nuclease-free water (Ambion). The mRNAs were diluted in nuclease-free water (Ambion) with $0.05 \%$ Fast Green vital dye (MilliporeSigma) at a concentration of $60 \mathrm{ng} / \mu \mathrm{l}$ (FUS), $25 \mathrm{ng} / \mu \mathrm{l}$ (TDP-43), and $100 \mathrm{ng} / \mu \mathrm{l}$ (SOD) were pulse-injected into 1- to 2-cell stage embryos using a Picospritzer III pressure ejector.

Drug screening protocol. Zebrafish embryos at 24 hours after fertilization (hpf) were placed in individual wells in a 24-well plate and were treated overnight with compounds in embryo medium (E3). Behavioral touch responses were then assessed at 52-56 hpf. Briefly, zebrafish larvae were touched lightly at the level of the tail with a pair of blunt forceps, and their locomotor behavior was recorded with a Grasshopper 2 Camera (Point Grey Research) at $30 \mathrm{~Hz}$. The movies were then analyzed using the manual tracking plugin of Image J 1.45r software (NIH), and the swim duration, swim distance, and maximum swim velocity of the fish were calculated.

Motor axon and NMJ morphology. Immunohistochemical analyses were performed on 48 hpf zebrafish to visualize axonal projections of motor neurons, as well as NMJ pre- and postsynaptic structures. Briefly, animals were fixed in $4 \%$ paraformaldehyde overnight at $4^{\circ} \mathrm{C}$. After fixation, the larvae were rinsed several times (1 hour) with PBS and then incubated in PBS containing $1 \mathrm{mg} / \mathrm{ml}$ collagenase (20 minutes) to remove skin. The collagenase was washed off with PBS (1 hour), and the larvae were incubated in PBST (PBS with Triton X-100) containing $10 \mathrm{mg} / \mathrm{ml}$ sulforhodamine conjugated $\alpha$ BTX (30 minutes). The larvae were rinsed several times with PBST (30 minutes) and then incubated in freshly prepared block solution containing primary antibody ZNP-1 (1:100, catalog ZNP1DSHB, Developmental Studies Hybridoma 
Bank) overnight at $4^{\circ} \mathrm{C}$. Following this, larvae were incubated in block solution containing a secondary antibody (Alexa fluor 488, 1:1,000, catalog A-21042, Invitrogen) for 6 hours at $4^{\circ} \mathrm{C}$ before being mounted on a glass slide in $70 \%$ glycerol. Fluorescent images of fixed embryos were taken using a Quorum Technologies spinning-disk confocal microscope mounted on an upright Olympus BX61W1 fluorescence microscope equipped with an Hamamatsu ORCA-ER camera. Image acquisition was performed with Volocity software (PerkinElmer). All chemicals were purchased from MilliporeSigma.

Electrophysiological recordings. Paired motoneuron/muscle recordings were performed following procedures previously described (14). Briefly, paired motor motoneuron/muscle recordings were obtained by perfusing collagenase $(1 \mathrm{mg} / \mathrm{ml})$ over the preparation for 10 minutes. This allowed for partial digestion before muscle fibers overlying the spinal cord were removed by aspiration to expose the spinal cord while leaving the ventral root and deeper muscle cells intact. Somites 13-16 were selected for recording. Patchclamp electrodes (7-9 M $\Omega$ ) were filled with the following intracellular solution (in $\mathrm{mM}$ ): $116 \mathrm{~K}$-gluconate; $16 \mathrm{KCl} ; 2 \mathrm{MgCl}_{2} ; 10 \mathrm{HEPES} ; 10$ EGTA adjusted to $\mathrm{pH} 7.2$, and $290 \mathrm{mOsm}$. The caudal and primary (CaP) motoneuron was selected because of its size and projection pattern to intact ventral regions of the trunk musculature. Motoneuron action potentials (APs) were elicited by a train of $200 \mathrm{pA}, 2 \mathrm{~ms}$ current steps into the motoneuron soma in the current-clamp mode at $30 \mathrm{~Hz}$ for $10 \mathrm{~s}$. Muscle patch-clamp electrodes (4-5 $\mathrm{M} \Omega$ ) were filled using the same intracellular solution, except $1 \mu \mathrm{M}$ QX-314 (MilliporeSigma) was added to the patch solution to block voltage-gated sodium currents and muscle contractions.

Calcium currents were recorded in the whole-cell configuration with an Axopatch 200B patch-clamp amplifier (Molecular Devices), and series resistance was compensated by at least $85 \%$ using the amplifier's compensation circuitry. Voltage protocol generation and data acquisition were performed using the pCLAMP10 software (Molecular Devices). Patch electrodes with resistances of 7-9 M $\Omega$ contained $110 \mathrm{mM}$ Cs-gluconate, $10 \mathrm{mM} \mathrm{CsCl}, 1 \mathrm{mM} \mathrm{CaCl}$, $1 \mathrm{mM} \mathrm{Mg-ATP,} 1 \mathrm{mM}$ EGTA, and $10 \mathrm{mM}$ HEPES, $\mathrm{pH}$ 7.4. The bath solution contained $100 \mathrm{mM} \mathrm{NaCl}, 2 \mathrm{mM} \mathrm{CsCl}, 1 \mathrm{mM} \mathrm{MgCl}_{2}, 5 \mathrm{mM} \mathrm{CaCl}_{2}, 10 \mathrm{mM}$ Glucose, $10 \mathrm{mM}$ HEPES, $20 \mathrm{mM}$ tetraethylammonium (TEA), $1 \mathrm{mM}$ 4-aminopyridine (4-AP), and $1 \mu \mathrm{M}$ tetrodotoxin (TTX), $\mathrm{pH} 7.8$.

\section{Mouse studies}

Genetic model. Mice overexpressing the G37R mutant human SOD1 transgene were obtained from the Jackson Laboratory and bred at our facility at Université de Montréal with females on a C57BL/6 background. Heterozygotes mice from the original line 29 usually overexpress 2-5 copies of the mutant form of the protein in the brain and spinal cord (29). This line of mutant mice has been characterized to develop a slow disease progression and shows symptoms comparable with ALS in humans, with onset of hindlimb paralysis starting after more than 1 year of age. Experiments were performed on $S O D 1^{G 37 R}$ mice and their WT littermates. PCR amplification of the human SOD1 gene was used to identify mutant mice by taking a small tail biopsy sample at around 3 weeks of age (weaning time). To be able to investigate the presymptomatic period, 5- to 6-month-old mice (P150-180) were used, and 12- to 13-month-old mice (P380-400) were used to assess the period prior to disease onset. Recordings were unfortunately not possible after disease onset because of the reduced muscle quality due to disease progression that did not allow us to perform recordings properly.

Nerve-muscle preparations. Nerve-muscle preparations of the EDL were carefully dissected in an oxygenated ( $95 \% \mathrm{O}_{2}, 5 \% \mathrm{CO}_{2}$ ) Ringer's Solution (in mM): $110 \mathrm{NaCl}, 5 \mathrm{KCl}, 1 \mathrm{MgCl}_{2}, 25 \mathrm{NaHCO}_{3}, 2 \mathrm{CaCl}_{2}, 11$ glucose, 0.3 glutamic acid, 0.4 glutamine, $5 \mathrm{BES}, 0.036$ choline chloride, and $4.34 \times 10^{-7}$ cocarboxylase. After dissection, muscles were pinned in a sylgard-coated recording chamber containing the Ringer's Solution. In order to block muscle contractions during synaptic recordings, nerve-muscle preparations were constantly perfused with an oxygenated $\left(95 \% \mathrm{O}_{2}, 5 \% \mathrm{CO}_{2}\right)$ modified Ringer's solution with low $\mathrm{Ca}^{2+}(1$ $\mathrm{mM})$ and high $\mathrm{Mg}^{++}(6.4 \mathrm{mM})$. All experiments were then performed at $28^{\circ} \mathrm{C}-30^{\circ} \mathrm{C}$.

Electrophysiological recordings of NMJ synaptic transmission. Evoked synaptic activity in muscles was recorded in response to a suprathreshold stimulation of the tibial nerve $(2 \times-5 \times)$ using a suction electrode filled with the Ringer's solution. Intracellular synaptic recordings of EPPs and mEPPs were performed using sharp glass microelectrodes (35-60 M $\Omega$, filled with $3 \mathrm{M} \mathrm{KCl}$ ). Muscle fibers were impaled around $50 \mathrm{~nm}$ from the adjacent NMJ. Synaptic transmission (EPP and mEPP) signals were first amplified using an HS-2A Headstage by Axon Instruments, then amplified again $(1,000 \times)$ and finally filtered with a low-pass filter (2 $\mathrm{KHz}$ ) by a Warner Instruments DC amplifier. A national Instruments BNC 2110 board was used to digitize the recordings that were acquired with WinWCP software (provided by John Dempster, Strathclyde University, Strathclyde, United Kingdom). 
To evaluate basal spontaneous activity of the muscle, a 5- to 10-minute recording period without any motor nerve stimulation was first performed to record mEPP amplitude and frequency. The frequency of miniature events was obtained by counting the number of mEPPs occurring over a period of at least 5 minutes, with the frequency being calculated in $\mathrm{mEPP} /$ seconds.

Synaptic strength of the recorded NMJs was assessed using the paired-pulsed facilitation ratio (PPF) and the QC. The stimulation protocol to elicit evoked activity and calculate synaptic strength consisted of 2 nerve stimuli $(0.1 \mathrm{~ms}$ duration) at $0.2 \mathrm{~Hz}$, with a 10 -ms interval between stimuli. PPF was calculated using the ratio of the mean amplitude of the second EPPs divided by the mean amplitude of the first EPP, including failures, over a period of 10 minutes. QC was calculated using the method of mEPPs: mean amplitude of the first EPPs/mean amplitude of mEPPs. Failures were also included in the QC calculation.

Pimozide effect on neurotransmission. To investigate the effect of the pimozide on synaptic transmission at the $S O D 1^{G 37 R}$ mice NMJ, bath applications of the drug were performed on nerve muscle preparations after recording of basal transmission for 20-25 minutes. The hydrophobic pimozide powder was first dissolved in DMSO and then incorporated to the modified Ringer's solution at a concentration of $5 \mu \mathrm{M}(0.16 \%$ DMSO). Preparations were continuously perfused for a period of 20-30 minutes to allow the drug to exert its effect the NMJ and to assess the impact on neurotransmission. Spontaneous activity and evoked activity were then recorded again following the same protocol previously described for a period of $20-45$ minutes.

\section{Clinical studies}

The clinical trial protocol was approved by the University of Calgary Conjoint Health Research Ethics Board (CHREB) and Health Canada. All study visits were conducted at the Department of Clinical Neurosciences (5th Floor), South Health Campus, Calgary, Alberta, Canada.

Randomization and masking. Twenty-five subjects meeting all inclusion and no exclusion criteria were randomized (Selection criteria summarized in Table 1). Subjects were block randomized (block size of 5 subjects). Subjects received either study drug or placebo.

Study drug (pimozide) was initiated at $1 \mathrm{mg}$ twice daily and then increased by $1 \mathrm{mg}$ twice daily every 5 days, to $3 \mathrm{mg}$ twice daily (Group 1; 10 subjects, $6 \mathrm{mg}$ /day) and $5 \mathrm{mg}$ twice daily (Group 2; 10 subjects; $10 \mathrm{mg} /$ day). The maximum dose was administered for 4 weeks. In Group 3 (5 subjects), subjects were provided placebo tablets. Study drug or placebo was titrated by reducing the dose by $1 \mathrm{mg}$ twice daily every day to full discontinuation over 5 days. Due to poor tolerability at doses of pimozide above $4 \mathrm{mg}$, the protocol was amended to reduce the doses to $4 \mathrm{mg} /$ day and $2 \mathrm{mg} /$ day, as subjects tolerated those doses in the uptitration phase and following reductions from higher doses. As a result of this change in protocol, we defined low dose as $2 \mathrm{mg} /$ day and high dose as $4 \mathrm{mg} /$ day or higher.

Adverse events and concomitant medications were collected every visit during the trial. The MedDRA adverse event dictionary was used (http://www.medra.org). ECG was performed by cardiodiagnostic technicians at screen, complete titration, midstudy, final outcomes, and end-of-study visits. Blood work was performed to exclude medical conditions that could meet exclusion criteria (screen) and at final-outcomes visit for safety. Neurological examination was performed by neurologists at screen, final-outcome, and final visits. Physical examinations were performed at screen and final visits.

Subjects developing adverse effects at any dose of the active or control drug had the dose reduced and were rechallenged in cases determined by the principal investigator that it was safe to do so. Blinding of investigators, assessors, and subjects was maintained throughout the study. An unblinded study physician monitored adverse effects throughout the study.

RNS. The procedure was performed using the Cadwell Sierra Electromyographic System (Cadwell Laboratories) using a standard and previously published technique (48) at screen, randomization (day 1), final outcomes (day $51 \pm 3$ days), and end-of-study visits. An electrophysiology technician blinded to treatment allocation and previous RNS test results performed the test. The desired nerve-muscle pairs were stimulated (bilateral median to abductor pollicis brevis, ulnar nerve to abductor digiti minimi, and left accessory nerve to trapezius). Nerve muscle pairs with CMAPs less than $1 \mathrm{mV}$ were excluded from further testing. A 3-Hz train of 10 stimuli was performed and percent decrement between 1 st and 4 th stimulus was calculated. A blinded neurologist/neurophysiologist reviewed all studies, and those determined to be technically unsound were excluded from the analysis. 
Table 1. Eligibility criteria for pimozide phase IIb trial

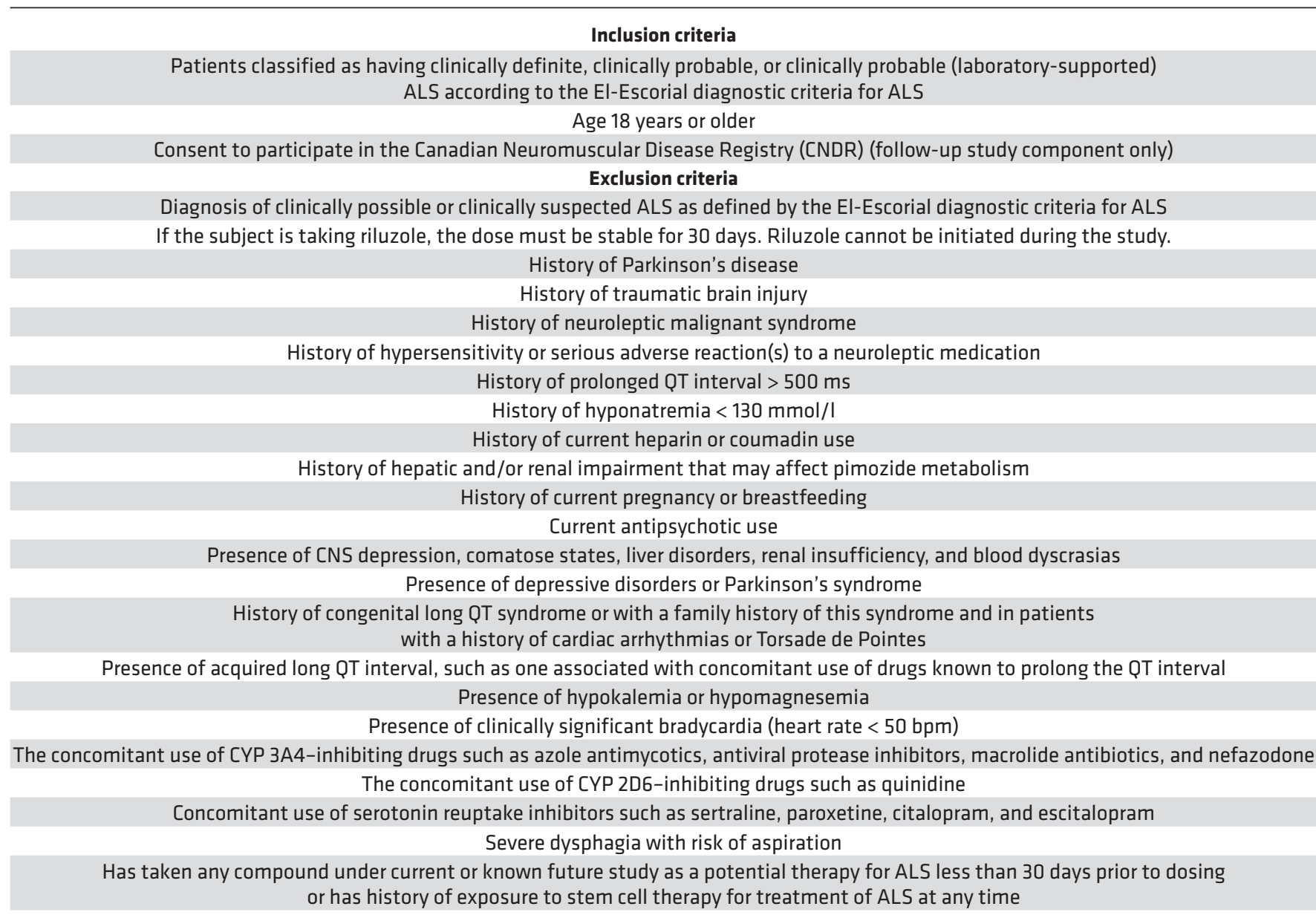

Twenty-five subjects meeting all inclusion and no exclusion criteria were invited to participate in the study. QT, the time between the start of the Q wave and the end of the T wave in the heart's electrical cycle.

SVC. SVC tests were administered at screen, randomization, final-outcome, and end-of-study visits performed using NDD EasyOne Spirometer System accessories (NDD Medical Technologies Inc.). The EasyOne Spirometer System does not require calibration (as per NDD). Three to 5 SVC tests were performed. The highest result was accepted after 3 if variability was less than or equal to $10 \%$.

$A L S F R S-R$. The ALSFRS-R was administered to subjects at randomization, final-outcome, and endof-study visits.

\section{MRC sum score}

An MRC sum score was performed at screen, final-outcome, and end-of-study visits, scoring a MRC muscle power rating (0-5) for 11 muscles on each side for a maximum total score out of 110 at each visit. A MRC sum score change score was calculated subtracting the MRC sum score at the end-of-treatment visit from the score at the randomization visit.

EDC system and randomization. We used a customized Clincapture EDC system (Clincapture) for data capture and randomization.

Statistics. For worms, survival curves were generated for the drug screen and synaptic transmission tests and compared using the Log-rank (Mantel-Cox) test. All values are presented in mean $\pm \mathrm{SEM}$, and $P<$ 0.05 was considered statistically significant. For the neurodegeneration assays, the mean and \pm SEM were calculated for each trial, and 2-tailed $t$ tests were used for statistical analysis.

For zebrafish, all data values are given as mean \pm SEM, and $P<0.05$ was considered statistically significant. Significance was determined using one-way ANOVA and Fisher LSD tests for normally 
distributed and equal variance data; Kruskal-Wallis ANOVA and Dunn's method of comparison were used for nonnormal distributions.

For mice, all data values are given as mean \pm SEM. Significance was determined using one-way ANOVA and Fisher LSD tests for normally distributed and equal variance data. Kruskal-Wallis ANOVA and Dunn's method of comparison were used for nonnormal distributions, and $P<0.05$ was considered statistically significant.

For clinical studies, all analyses reported were performed according to the intention-to-treat principle. Per-protocol analyses were also performed but are not reported. Primary outcome measure analyses were performed using nonparametric analyses, including Wilcoxon rank-sum test for change scores between end-of-treatment and randomization visits, and Kruskal-Wallis $\mathrm{H}$ test for comparison between multiple groups with correction for repeated measures. $P<0.05$ was considered statistically significant. Additional linear mixed model regression analysis was performed for analysis of decremental response change from end of treatment based on actual dose of pimozide.

\section{Study approval}

The present studies in animals and humans were reviewed and approved by IRBs of the CRCHUM and University of Montreal (Montreal, Quebec, Canada) Ethics Committee, ICM Ethics Committee (Paris, France), St. Michael's Hospital Ethics Committee (Toronto, Ontario, Canada), and the University of Calgary Conjoint Health Research Ethics Committee (Calgary, Alberta, Canada) and Health Canada. Subjects provided informed consent prior to their participation in the study. All experiments were performed in compliance with the guidelines of the Canadian Council for Animal Care.

\section{Author Contributions}

Conceptualization contributed by PD; methodology contributed by SAP, DA, GABA, RR, JAP, LZ, LK, and PD; investigation contributed by SAP, DA, JM, ET, JP, GABA, ALF, CM, ML, SC, XYW, VR, JI, LZ, JPJ, $\mathrm{EK}$, and RR; writing contributed by SAP, JAP, LK, and PD; funding acquisition contributed by PD, JAP, and LK; visualization contributed by SAP, JAP, and PD; supervision contributed by PD, JAP, RR, and LK.

\section{Acknowledgments}

We express our heartfelt thanks to the patients who participated in this study. The authors would also like to thank Marina Drits and Guy Laliberté for excellent care of the zebrafish. We thank Guy Rouleau for introducing many of us to ALS research and for providing the TARDBP, FUS, and SOD1 clones used; Randall Peterson for guiding us in the design of the chemical genetic screens; and Terry Snutch for insights to pimozide pharmacology. SAP was supported by Canadian Institutes of Health Research (CIHR) and the Réseau Médical de Génétique Appliquée fellowships. GABA was supported by a CIHR fellowship. PD and JPJ were supported by Canada Research Chairs, and JKI is a New York Stem Cell Foundation Robertson Investigator. The biological studies were supported by Genome Québec (PD and EK), the Frick Foundation for ALS Research (PD, AP), the US Department of Defense CDMRP/ALSRP (PD, JAP, EK and JPJ), Brain Canada and OCE-CQDM (PD and X-YW), the Weston Brain Institute (PD, GABA, RR), the Muscular Dystrophy Association USA (JAP), the CIHR (JAP, PD, RR), the ALS Association (PD, AP, X-YW, RR), and NIH grants R00NS077435 and R01NS097850 (JKI). The clinical study was funded through a generous donation from the Quirk family (Calgary, Alberta, Canada) and a grant from the Hotchkiss Brain Institute and the Clinical Research Unit at the University of Calgary. Patient study visit space and support was provided by South Health Campus, Calgary, Alberta. Statistical analysis support was provided by the Clinical Research Unit, Faculty of Medicine, University of Calgary. SAP was supported by Canadian Institutes of Health Research (CIHR) and the Réseau Médical de Génétique Appliquée fellowships. GABA was supported by a CIHR fellowship. JAP was supported by a FRQS Research Scholar Award, PD and JPJ were supported by Canada Research Chairs, and JKI is a New York Stem Cell Foundation Robertson Investigator.

Address correspondence to: Pierre Drapeau, Centre de recherche du CHUM, Tour Viger R09-482, 900, rue Saint-Denis, Montreal, H2X 0A9, Canada, Quebec. Phone: 1.514.890.8000; Email: p.drapeau@umontreal.ca. 
1. Glicksman MA. The preclinical discovery of amyotrophic lateral sclerosis drugs. Expert Opin Drug Discov. 2011;6(11):1127-1138.

2. Bensimon G, Lacomblez L, Meininger V. A controlled trial of riluzole in amyotrophic lateral sclerosis. ALS/Riluzole Study Group. NEngl J Med. 1994;330(9):585-591.

3. Abe K, et al. Confirmatory double-blind, parallel-group, placebo-controlled study of efficacy and safety of edaravone (MCI-186) in amyotrophic lateral sclerosis patients. Amyotroph Lateral Scler Frontotemporal Degener. 2014;15(7-8):610-617.

4. Mulder DW, Lambbert EH, Eaton LM. Myasthenic syndrome in patients with amyotrophic lateral sclerosis. Neurology. 1959;9:627-631.

5. Hodes R. Electromyographic study of defects of neuromuscular transmission in human poliomyelitis. Arch Neurol Psychiatry. 1948;60(5):457-473.

6. Killian JM, Wilfong AA, Burnett L, Appel SH, Boland D. Decremental motor responses to repetitive nerve stimulation in ALS. Muscle Nerve. 1994;17(7):747-754.

7. Yamashita S, et al. Significant CMAP decrement by repetitive nerve stimulation is more frequent in median than ulnar nerves of patients with amyotrophic lateral sclerosis. Muscle Nerve. 2012;45(3):426-428.

8. Maselli RA, et al. Neuromuscular transmission in amyotrophic lateral sclerosis. Muscle Nerve. 1993;16(11):1193-1203.

9. Dadon-Nachum M, Melamed E, Offen D. The "dying-back" phenomenon of motor neurons in ALS. J Mol Neurosci. 2011;43(3):470-477.

10. Fischer LR, et al. Amyotrophic lateral sclerosis is a distal axonopathy: evidence in mice and man. Exp Neurol. 2004;185(2):232-240.

11. Murray LM, Talbot K, Gillingwater TH. Review: neuromuscular synaptic vulnerability in motor neurone disease: amyotrophic lateral sclerosis and spinal muscular atrophy. Neuropathol Appl Neurobiol. 2010;36(2):133-156.

12. Zhou H, et al. Transgenic rat model of neurodegeneration caused by mutation in the TDP gene. PLoS Genet. 2010;6(3):e1000887.

13. Maselli RA, et al. Neuromuscular transmission in amyotrophic lateral sclerosis. Muscle Nerve. 1993;16(11):1193-1203

14. Armstrong GA, Drapeau P. Calcium channel agonists protect against neuromuscular dysfunction in a genetic model of TDP-43 mutation in ALS. J Neurosci. 2013;33(4):1741-1752.

15. Armstrong GA, Drapeau P. Loss and gain of FUS function impair neuromuscular synaptic transmission in a genetic model of ALS. Hum Mol Genet. 2013;22(21):4282-4292.

16. Mohajeri MH, Figlewicz DA, Bohn MC. Intramuscular grafts of myoblasts genetically modified to secrete glial cell line-derived neurotrophic factor prevent motoneuron loss and disease progression in a mouse model of familial amyotrophic lateral sclerosis. Hum Gene Ther. 1999;10(11):1853-1866.

17. Miller RG, et al. A placebo-controlled trial of recombinant human ciliary neurotrophic (rhCNTF) factor in amyotrophic lateral sclerosis. rhCNTF ALS Study Group. Ann Neurol. 1996;39(2):256-260.

18. Borasio GD, et al. A placebo-controlled trial of insulin-like growth factor-I in amyotrophic lateral sclerosis. European ALS/ IGF-I Study Group. Neurology. 1998;51(2):583-586.

19. Acsadi G, et al. Increased survival and function of SOD1 mice after glial cell-derived neurotrophic factor gene therapy. Hum Gene Ther. 2002;13(9):1047-1059.

20. Swarup V, et al. Pathological hallmarks of amyotrophic lateral sclerosis/frontotemporal lobar degeneration in transgenic mice produced with TDP-43 genomic fragments. Brain. 2011;134(Pt 9):2610-2626.

21. Patten SA, Parker JA, Wen XY, Drapeau P. Simple animal models for amyotrophic lateral sclerosis drug discovery. Expert Opin Drug Discov. 2016;11(8):797-804.

22. Vaccaro A, Tauffenberger A, Aggad D, Rouleau G, Drapeau P, Parker JA. Mutant TDP-43 and FUS cause age-dependent paralysis and neurodegeneration in C. elegans. PLoS ONE. 2012;7(2):e31321.

23. Kabashi E, et al. Gain and loss of function of ALS-related mutations of TARDBP (TDP-43) cause motor deficits in vivo. Hum Mol Genet. 2010;19(4):671-683.

24. Vaccaro A, et al. Pharmacological reduction of ER stress protects against TDP-43 neuronal toxicity in vivo. Neurobiol Dis. 2013;55:64-75.

25. Vaccaro A, et al. Methylene blue protects against TDP-43 and FUS neuronal toxicity in C. elegans and D. rerio. PLoS ONE. 2012;7(7):e42117.

26. Kabashi E, et al. FUS and TARDBP but not SOD1 interact in genetic models of amyotrophic lateral sclerosis. PLoS Genet 2011;7(8):e1002214

27. Mahoney TR, Luo S, Nonet ML. Analysis of synaptic transmission in Caenorhabditis elegans using an aldicarb-sensitivity assay. Nat Protoc. 2006;1(4):1772-1777.

28. Buss RR, Drapeau P. Synaptic drive to motoneurons during fictive swimming in the developing zebrafish. JNeurophysiol. 2001;86(1):197-210.

29. Wong PC, et al. An adverse property of a familial ALS-linked SOD1 mutation causes motor neuron disease characterized by vacuolar degeneration of mitochondria. Neuron. 1995;14(6):1105-1116.

30. Pun S, Santos AF, Saxena S, Xu L, Caroni P. Selective vulnerability and pruning of phasic motoneuron axons in motoneuron disease alleviated by CNTF. Nat Neurosci. 2006;9(3):408-419.

31. Frey D, Schneider C, Xu L, Borg J, Spooren W, Caroni P. Early and selective loss of neuromuscular synapse subtypes with low sprouting competence in motoneuron diseases. J Neurosci. 2000;20(7):2534-2542.

32. Tremblay E, Martineau É, Robitaille R. Opposite Synaptic Alterations at the Neuromuscular Junction in an ALS Mouse Model: When Motor Units Matter. J Neurosci. 2017;37(37):8901-8918.

33. Santi CM, et al. Differential inhibition of T-type calcium channels by neuroleptics. J Neurosci. 2002;22(2):396-403

34. Enyeart JJ, Biagi BA, Mlinar B. Preferential block of T-type calcium channels by neuroleptics in neural crest-derived rat and human C cell lines. Mol Pharmacol. 1992;42(2):364-372.

35. Enyeart JJ, Dirksen RT, Sharma VK, Williford DJ, Sheu SS. Antipsychotic pimozide is a potent Ca2+ channel blocker in heart. Mol Pharmacol. 1990;37(5):752-757.

36. Bancila M, Copin JC, Daali Y, Schatlo B, Gasche Y, Bijlenga P. Two structurally different T-type Ca 2+ channel inhibitors, mibefradil and pimozide, protect CA1 neurons from delayed death after global ischemia in rats. Fundam Clin Pharmacol. 
2011;25(4):469-478.

37. Szczudlik A, Tomik B, Słowik A, Kasprzyk K. [Assessment of the efficacy of treatment with pimozide in patients with amyotrophic lateral sclerosis. Introductory notes]. Neurol Neurochir Pol. 1998;32(4):821-829.

38. Shapiro AK, Shapiro E, Fulop G. Pimozide treatment of tic and Tourette disorders. Pediatrics. 1987;79(6):1032-1039.

39. Pinder RM, Brogden RN, Swayer R, Speight TM, Spencer R, Avery GS. Pimozide: a review of its pharmacological properties and therapeutic uses in psychiatry. Drugs. 1976;12(1):1-40.

40. Rondanin R, et al. Inhibition of activated STAT5 in Bcr/Abl expressing leukemia cells with new pimozide derivatives. Bioorg Med Chem Lett. 2014;24(18):4568-4574.

41. Zhou W, et al. The antipsychotic drug pimozide inhibits cell growth in prostate cancer through suppression of STAT3 activation. Int J Oncol. 2016;48(1):322-328.

42. Renna M, Jimenez-Sanchez M, Sarkar S, Rubinsztein DC. Chemical inducers of autophagy that enhance the clearance of mutant proteins in neurodegenerative diseases. J Biol Chem. 2010;285(15):11061-11067.

43. Eisen A, Kuwabara S. The split hand syndrome in amyotrophic lateral sclerosis. J Neurol Neurosurg Psychiatr. 2012;83(4):399-403.

44. Devine MS, Kiernan MC, Heggie S, McCombe PA, Henderson RD. Study of motor asymmetry in ALS indicates an effect of limb dominance on onset and spread of weakness, and an important role for upper motor neurons. Amyotroph Lateral Scler Frontotemporal Degener. 2014;15(7-8):481-487.

45. Stommel EW, Graber D, Montanye J, Cohen JA, Harris BT. Does treating schizophrenia reduce the chances of developing amyotrophic lateral sclerosis? Med Hypotheses. 2007;69(5):1021-1028.

46. McLaughlin RL, et al. Genetic correlation between amyotrophic lateral sclerosis and schizophrenia. Nat Commun. 2017;8:14774

47. Kimmel CB, Ballard WW, Kimmel SR, Ullmann B, Schilling TF. Stages of embryonic development of the zebrafish. Dev Dyn. 1995;203(3):253-310.

48. Preston D, Shapiro B. Electromyography Neuromuscular Disorders: Clinical-Electrophysiologic Correlations. Philadelphia, PA: Elsevier: 2013 